Título artículo / Títol article:

Application of gas chromatography-(triple quadrupole) mass spectrometry with atmospheric pressure chemical ionization for the determination of multiclass pesticides in fruits and vegetables

Autores / Autors

Cherta Cucala, Laura ; Portolés Nicolau, Tania ; Beltrán Arandes, Joaquim ; Pitarch Arquimbau, María Elena ; Mol, Johannes G.J. ; Hernández Hernández, Félix

Revista:

Journal of Chromatography A Volume 1314, November 2013

Versión / Versió:

Preprint de l'autor

Cita bibliográfica / Cita bibliogràfica (ISO 690):

url Repositori UJI:

http://hdl.handle.net/10234/92971 


\title{
Application of gas chromatography-(triple quadrupole) mass spectrometry with atmospheric pressure chemical ionization for the determination of multiclass pesticides in fruits and vegetables
}

\author{
Laura Cherta ${ }^{a}$, Tania Portolésa,b, Joaquim Beltrana, Elena Pitarcha, Johannes G.J. Molb, Félix \\ Hernández \\ aResearch Institute for Pesticides and Water, University Jaume I, Castellón, Spain. \\ bRIKILT Institute of Food Safety, Wageningen University and Research Centre, Wageningen, \\ The Netherland
}

\begin{abstract}
A multi-residue method for the determination of 142 pesticide residues in fruits and vegetables has been developed using a new atmospheric pressure chemical ionization (APCI) source for coupling gas chromatography (GC) to tandem mass spectrometry (MS). Selected reaction monitoring (SRM) mode has been applied, acquiring three transitions for each compound. In contrast to the extensive fragmentation typically obtained in classical electron ionization (EI), the soft APCI ionization allowed the selection of highly abundant protonated molecules $\left([\mathrm{M}+\mathrm{H}]^{+}\right)$as precursor ions for most compounds. This was favorable for both sensitivity and selectivity. Validation of the method was performed in which both quantitative and qualitative parameters were assessed using orange, tomato and carrot samples spiked at two levels, 0.01 and $0.1 \mathrm{mg} / \mathrm{kg}$. The QuEChERS method was used for sample preparation, followed by a 10-fold dilution of the final acetonitrile extract with a mixture of hexane and acetone. Recovery and precision were satisfactory in the three matrices, at both concentration levels. Very low limits of detection (down $0.01 \mathrm{~g} / \mathrm{kg}$ for the most sensitive compounds) were achieved. Ion ratios were consistent and identification according to EU criteria was possible in $80 \%(0.01 \mathrm{mg} / \mathrm{kg})$ to $96 \%(0.1 \mathrm{mg} / \mathrm{kg})$ of the pesticide/matrix combinations. The method was applied to the analysis of various fruits and vegetables from the Mediterranean region of Spain.
\end{abstract}

\section{Keywords}

Atmospheric pressure chemical ionization; Gas chromatography; Tandem mass spectrometry; Triple quadrupole; Pesticides; Fruit and vegetable analysis, validation, identification 


\section{INTRODUCTION}

The control of pesticide residues in food commodities is a requirement to verify compliance with regulatory limits set by the European Commission (EC 396/2005) to ensure good agricultural practice and food safety. Sensitive and robust analytical techniques are required that preferably cover various pesticide chemical classes with different physicochemical properties. A common analytical approach is to combine generic sample preparation techniques, with inherently low selectivity, with highly selective instrumental analysis.

The QuEChERS (Quick, Easy, Cheap, Effective, Rugged and Safe) procedure is a popular generic sample preparation method for the extraction of pesticides from fruits and vegetables. It involves a rapid extraction using acetonitrile $(\mathrm{MeCN})$ and a cleanup step based on dispersive-SPE (d-SPE) using a primary secondary amine (PSA) sorbent and anhydrous $\mathrm{MgSO}_{4}$ to remove water [1] and [2]. Numerous applications have been successfully validated for a large number of pesticides in a variety of complex matrices [3], [4] and [5].

For a major part of the pesticides, liquid chromatography combined with mass spectrometry is considered as the method of choice [6]. However, many pesticides are also amenable to gas chromatography (GC) coupled to mass spectrometry (MS) which makes it a valuable complementary technique, especially because it is the only option for certain pesticide classes and therefore has to be used anyway. Several quantitative applications have been described in literature using GC-MS with a single quadrupole analyzer operating in selected ion monitoring (SIM) [7], [8] and [9], especially for multi-residue analysis with a limited number of compounds. However, the determination of a larger number of analytes usually requires more selective techniques, as tandem mass spectrometry (MS/MS). The use of triple quadrupole (QqQ) working under selected reaction monitoring (SRM) improves selectivity, as well as sensitivity [10], [11], [12], [13] and [14].

In GC-MS/MS, electron ionization (EI) is by far the most widely used ionization technique because of its capability of ionizing virtually any organic compound. A rather strong fragmentation is inherent to EI. This is a disadvantage in GC-MS/MS because in many cases fragments have to be used as precursor ions which are then further fragmented to smaller product ions. This compromises both sensitivity and selectivity compared to LC- 
MS/MS where quasi molecular ions are obtained during ionization. Softer ionization modes such as chemical ionization (PCI, NCI) and supersonic molecular beam (cold EI) [15] are available for GC but these options have restrictions with respect to applicability and commercial availability, respectively. Atmospheric pressure chemical ionization (APCI), commonly used in LC-MS/MS, has been described as an alternative source for GC-MS and a way to couple GC to mass spectrometers initially developed for LC-MS [16] and [17]. Application studies including pharmaceutical development [18], profiling of phenolic compounds in oil [19], metabolic profiling [20] and pesticide residue analysis [21], most of them using GC-(APCI) TOF MS, can be found since 2009. Recently, we investigated the potential of APCI in GC-triple quadrupole MS for wide-scope pesticide residue analysis [22] and [23]. Compared to EI, little or no fragmentation occurs while compared to PCI/NCI the applicability to different classes of compounds was much wider. Besides the selectivity advantage arising from the ability to use the quasi-molecular ion as precursor ion, the sensitivity was also found to be substantially improved. This was partly due to the use of high-end MS/MS detectors normally used for LC-MS/MS (in fact, by changing the source, the same MS/MS could be coupled to either LC or GC).

In the previous papers the emphasis was on the potential, ionization mechanisms, and features of GC-(APCI) MS/MS. In this work the focus is on applicability for routine wide-scope multi-residue analysis of pesticides in fruits and vegetable matrices, with emphasis on quantitative and qualitative performance. A GC-(APCI) MS/MS method for simultaneous detection of 142 pesticides (around 48 of them non LC-amenable) was set up including three transitions for each compound. Using a QuEChERS method for sample preparation, the method was validated for three matrices (orange, tomato, carrot). Matrix effects, linearity, accuracy, limits of quantification (LOQs) and limits of detection (LODs) were established. With respect to identification, compliance of ion ratios with EU criteria was assessed. Real samples were analyzed to test the method applicability, including orange, tomato, carrot and also apple, lettuce and courgette. 


\section{EXPERIMENTAL}

\subsection{Reagents}

Pesticide standards were purchased from Dr. Ehrenstorfer (Augsburg, Germany). Stock standard solutions (around $500 \mathrm{~g} / \mathrm{mL}$ ) were prepared by dissolving reference standards in acetone and stored in a freezer at $20{ }^{\circ} \mathrm{C}$. Working standard mixtures were prepared by volume dilution of stock solutions in hexane for preparation of matrix-matched calibrants and in acetone for sample fortification.

Hexane, acetone, acetonitrile (MeCN), toluene, glacial acetic acid (HAc), anhydrous $\mathrm{MgSO}_{4}$ and anhydrous sodium acetate (NaAc) were purchased from Scharlab (Barcelona, Spain). Solvents used were of pesticide residue analysis or HPLC grade. Two types of $2 \mathrm{~mL}$ micro-centrifuge tubes for QuEChERS d-SPE containing $50 \mathrm{mg}$ PSA and $150 \mathrm{mg}$ anhydrous $\mathrm{MgSO} 4$, or $50 \mathrm{mg} \mathrm{PSA}, 150 \mathrm{mg}$ anhydrous $\mathrm{MgSO}_{4}$ and $50 \mathrm{mg} \mathrm{C} 18$, were obtained from Teknokroma (Barcelona, Spain).

\subsection{Sample material}

Three types of sample matrices were used in the validation study: orange, tomato and carrot. Blank samples, used for the matrix-matched calibration, sample fortification and quality control, were obtained from organic cultivars (pesticide free).

Three different varieties from each food commodity were analyzed to investigate the presence of pesticides, all of them purchased from local markets in the Castellón province (Spain).

Apple, lettuce and courgette samples, also purchased from local markets, were analyzed to extend the method applicability.

\subsection{Instrumentation}

Data were acquired using a GC system (Agilent 7890A, Palo Alto, CA, USA) equipped with an autosampler (Agilent 7693) and coupled to a triple quadrupole (QqQ) mass spectrometer (Xevo TQ-S, Waters Corporation, Manchester, UK), operating in APCI mode. A fused silica DB-5MS capillary column (length $30 \mathrm{~m} \times$ I.D. $0.25 \mathrm{~mm} \times$ film $0.25 \mathrm{~m}$ ) 
(J\&W Scientific, Folson, CA, USA) was used for GC separation. The injector was operated in splitless mode, injecting $1 \quad \mathrm{~L}$ at $280{ }^{\circ} \mathrm{C}$. The oven temperature was programmed as follows: $70{ }^{\circ} \mathrm{C}(1 \mathrm{~min}), 15{ }^{\circ} \mathrm{C} / \mathrm{min}$ to $150{ }^{\circ} \mathrm{C}$ and $10{ }^{\circ} \mathrm{C} / \mathrm{min}$ to $300{ }^{\circ} \mathrm{C}(3 \mathrm{~min})$. Helium was used as carrier gas in constant flow mode $(2 \mathrm{~mL} / \mathrm{min})$. A pulsed splitless injection was carried out using an initial pressure of $240 \mathrm{kPa}$, maintained for $1 \mathrm{~min}$, and then changed to a constant flow of $2 \mathrm{~mL} / \mathrm{min}$, which corresponded to a linear velocity of $52 \mathrm{~cm} / \mathrm{s}$. In the SRM method, automatic dwell time (values ranging from 3 to $63 \mathrm{~ms}$ ) was applied in order to obtain 15 points per peak.

The interface temperature was set to $310{ }^{\circ} \mathrm{C}$ using $\mathrm{N} 2$ as auxiliary gas at $250 \mathrm{~L} / \mathrm{h}$, a make-up gas at $300 \mathrm{~mL} / \mathrm{min}$ and cone gas at $170 \mathrm{~L} / \mathrm{h}$. The APCI corona discharge pin was operated at 1.8 A. The water used as modifier when working under proton-transfer conditions was placed in an uncapped vial, which was located within a holder placed in the source door.

Targetlynx (a module of MassLynx) was used to handle and process the acquired data.

\subsection{Sample treatment}

The QuEChERS procedure applied was that proposed in the AOAC official method 2007.01 [2]: $15 \mathrm{~g}$ of sample (previously homogenized in a food chopper) were weighted in a $50 \mathrm{~mL}$ polypropylene centrifuge tube, mixed with $15 \mathrm{~mL} \mathrm{MeCN}$ (with 1\% HAc) and shaken by hand for $30 \mathrm{~s}$. Then, $6 \mathrm{~g}$ anhydrous $\mathrm{MgSO}_{4}$ and $1.5 \mathrm{~g}$ anhydrous NaAc were added and immediately shaken vigorously by hand to prevent formation of $\mathrm{MgSO}_{4}$ agglomerates. Then, the tube was centrifuged at 3000 rpm for $2 \mathrm{~min}$.

For the cleanup step, $1 \mathrm{~mL}$ of the upper MeCN extract was transferred into a d-SPE tube containing $150 \mathrm{mg} \mathrm{MgSO} 4$ and $50 \mathrm{mg}$ PSA (or $150 \mathrm{mg} \mathrm{MgSO} 4,50 \mathrm{mg}$ PSA and $50 \mathrm{mg}$ C18 when oranges were extracted). The tubes were shaken on a Vortex for $30 \mathrm{~s}$ and centrifuged at $3000 \mathrm{rpm}$ for $2 \mathrm{~min}$. Finally, 50 L of the extract (acetonitrile) were transferred into a $2 \mathrm{~mL}$ vial and diluted with $300 \quad \mathrm{~L}$ of hexane and $150 \quad \mathrm{~L}$ of acetone.

Matrix-matched standards were prepared for each sample matrix as follows: after the cleanup step, 50 L of the MeCN extract obtained from a blank sample were mixed with $250 \mathrm{~L}$ of hexane, $150 \mathrm{~L}$ of acetone, and $50 \quad \mathrm{~L}$ of the pesticide standard solution in 
hexane at adequate concentration to obtain a calibration range of $0.1-100 \mathrm{ng} / \mathrm{mL}$ (corresponding to $1-1000 \mathrm{~g} / \mathrm{kg}$ in sample).

\subsection{Validation study}

The developed SRM method was validated using orange, carrot and tomato in order to evaluate linearity, recovery, precision, selectivity and LODs and LOQs.

Linearity was studied by injecting standards in hexane $(n=3)$ at eight concentration levels, $0.1,0.5,1,5,10,25,50$ and $100 \mathrm{ng} / \mathrm{mL}$, and was considered acceptable when regression coefficients were higher than 0.99 and residuals lower than $30 \%$.

Accuracy was estimated from recovery experiments, by analyzing six replicates spiked at two levels (0.01 and $0.1 \mathrm{mg} / \mathrm{kg}$ ). Precision was expressed as repeatability in terms of relative standard deviation $(\mathrm{RSD}, \%)(\mathrm{n}=6)$ calculated for each fortification level.

The LOQ was defined as the lowest concentration level validated with satisfactory values of recovery $(70-110 \%)$ and precision $(\mathrm{RSD}<20 \%)[24]$.

The LOD, defined as the concentration corresponding to a signal-to-noise ratio of three, was estimated from the chromatogram of the matrix-matched standards at the lowest calibration level used for each compound.

The selectivity of the method was evaluated by verification of the absence of interfering peaks at the retention time of each compound in blank samples for the acquired MS/MS transitions.

The ratio of each of the two qualifier ions relative to the quantifier (calculated by dividing the lower by the higher response) were used to verify compliance with EU criteria [24] of the pesticides in the spiked samples and to confirm peak identity in real samples. 


\section{RESULTS AND DISCUSSION}

\subsection{GC-(APCI) MS/MS optimization}

Optimization of the MS/MS conditions started by using pesticide standard solutions in hexane with the mass spectrometer operating in full scan mode to obtain the MS spectra. Experiments under N2 and proton transfer conditions (using water as modifier) were performed. The proton transfer mechanism revealed a notable tendency for the majority of the studied pesticides to be protonated, since the $[\mathrm{M}+\mathrm{H}]^{+}$was present for most compounds and frequently as the peak base of the spectrum, with very low fragmentation. Thus the use of water as modifier was considered for further experiments.

The cone voltage was studied in the range 5-40 $\mathrm{V}$ for all compounds and those values which result in higher sensitivity were selected for each pesticide (Table 1). The helium flow rate was set at a relatively high flow rate of $2 \mathrm{~mL} / \mathrm{min}$ since this was found to be beneficial for peak shape and analysis speed in an earlier work [23] using a GC(APCI) MS system.

To continue with MS/MS optimization, the base peak of the spectrum for each compound $\left([\mathrm{M}+\mathrm{H}]^{+}\right.$in most cases) was selected as precursor ion (in some cases, two different precursor ions were chosen). The main goal was to develop a SRM method with three MS/MS transitions (the most sensitive ones) in order to carry out a reliable quantification and identification of the pesticides detected in samples. The fragmentation pattern of the precursor ions was studied through product ion scan experiments at different collision energies (10, 20 and $30 \mathrm{eV})$ and again the most sensitive transitions were selected for the final SRM method. Table 1 shows the SRM transitions corresponding to both quantifier and the qualifier transitions monitored. 
Table 1. Experimental conditions of the optimized GC-(APCI)MS/MS method using water as modifier. Quantifier (Q) and qualifier (q) transitions.

\begin{tabular}{|c|c|c|c|c|c|c|}
\hline \multirow{2}{*}{$\frac{\mathrm{t}_{\mathrm{R}}(\min )}{6.2}$} & \multirow{2}{*}{$\frac{\text { Window (min) }}{6-7.75}$} & \multirow{2}{*}{$\begin{array}{l}\text { Compounds } \\
\text { Hexachlorobutadiene }\end{array}$} & \multicolumn{2}{|c|}{ SRM Transitions } & \multirow{2}{*}{$\begin{array}{c}\begin{array}{c}\text { Collision } \\
\text { energy }(\mathrm{eV})\end{array} \\
20\end{array}$} & \multirow{2}{*}{$\begin{array}{c}\begin{array}{c}\text { Cone voltage } \\
\text { (V) }\end{array} \\
30\end{array}$} \\
\hline & & & $\mathrm{Q}$ & $258>223$ & & \\
\hline & & & $\mathrm{q}_{1}$ & $258>141$ & 20 & \\
\hline & & & $\mathrm{q}_{2}$ & $258>188$ & 30 & \\
\hline \multirow[t]{3}{*}{6.45} & & Dichlorvos & $Q$ & $221>109$ & 20 & 10 \\
\hline & & & $\mathrm{q}_{1}$ & $221>127$ & 20 & \\
\hline & & & $\mathrm{q}_{2}$ & $221>145$ & 10 & \\
\hline \multirow[t]{3}{*}{6.95} & & Carbofuran & $\mathrm{Q}$ & $165>123$ & 10 & 20 \\
\hline & & & $\mathrm{q}_{1}$ & $165>105$ & 30 & \\
\hline & & & $\mathrm{q}_{2}$ & $165>137$ & 10 & \\
\hline \multirow[t]{3}{*}{8.19} & $7.75-8.8$ & Mevinphos & $\mathrm{Q}$ & $193>127$ & 10 & 30 \\
\hline & & & $\mathrm{q}_{1}$ & $225>127$ & 10 & \\
\hline & & & $\mathrm{q}_{2}$ & $225>193$ & 10 & \\
\hline \multirow[t]{3}{*}{8.45} & & Propham & $\mathrm{Q}$ & $138>120$ & 10 & 30 \\
\hline & & & $\mathrm{q}_{1}$ & $138>77$ & 30 & \\
\hline & & & $\mathrm{q}_{2}$ & $138>92$ & 10 & \\
\hline \multirow[t]{3}{*}{9} & $8.8-9.95$ & Carbaryl & $\mathrm{Q}$ & $145>117$ & 20 & 10 \\
\hline & & & $\mathrm{q}_{1}$ & $145>115$ & 20 & \\
\hline & & & $\mathrm{q}_{2}$ & $144>115$ & 20 & \\
\hline \multirow[t]{3}{*}{9.1} & & 2-Phenylphenol & $\mathrm{Q}$ & $171>153$ & 20 & 10 \\
\hline & & & $\mathrm{q}_{1}$ & $171>151$ & 30 & \\
\hline & & & $\mathrm{q}_{2}$ & $171>152$ & 30 & \\
\hline \multirow[t]{3}{*}{9.21} & & Pentachlorobenzene & $\mathrm{Q}$ & $248>213$ & 30 & 40 \\
\hline & & & $\mathrm{q}_{1}$ & $248>142$ & 30 & \\
\hline & & & $\mathrm{q}_{2}$ & $248>178$ & 30 & \\
\hline \multirow[t]{3}{*}{9.3} & & Molinate & $\mathrm{Q}$ & $188>126$ & 10 & 20 \\
\hline & & & $\mathrm{q}_{1}$ & $188>98$ & 20 & \\
\hline & & & $\mathrm{q}_{2}$ & $188>160$ & 10 & \\
\hline \multirow[t]{3}{*}{10.02} & $9.95-10.3$ & Propoxur & $\mathrm{Q}$ & $210>111$ & 10 & 10 \\
\hline & & & $\mathrm{q}_{1}$ & $210>168$ & 10 & \\
\hline & & & $\mathrm{q}_{2}$ & $210>135$ & 10 & \\
\hline \multirow[t]{3}{*}{10.05} & & Propachlor & $\mathrm{Q}$ & $212>170$ & 20 & 30 \\
\hline & & & $\mathrm{q}_{1}$ & $212>94$ & 30 & \\
\hline & & & $\mathrm{q}_{2}$ & $212>106$ & 30 & \\
\hline \multirow[t]{3}{*}{10.12} & & Demeton-s-methyl & $\mathrm{Q}$ & $143>111$ & 10 & 20 \\
\hline & & & $\mathrm{q}_{1}$ & $143>125$ & 10 & \\
\hline & & & $\mathrm{q}_{2}$ & $143>127$ & 10 & \\
\hline 10.14 & & Diphenylamine & $\mathrm{Q}$ & $170>93$ & 30 & 40 \\
\hline & & & $\mathrm{q}_{1}$ & $170>152$ & 20 & \\
\hline & & & $\mathrm{q}_{2}$ & $170>153$ & 20 & \\
\hline 10.39 & $10.2-10.8$ & Atrazine deisopropyl & $\mathrm{Q}$ & $174>132$ & 20 & 30 \\
\hline & & & $\mathrm{q}_{1}$ & $174>104$ & 30 & \\
\hline & & & $\mathrm{q}_{2}$ & $174>146$ & 20 & \\
\hline 10.4 & & Chlorpropham & $\mathrm{Q}$ & $172>154$ & 10 & 40 \\
\hline & & & $\mathrm{q}_{1}$ & $172>111$ & 20 & \\
\hline & & & $\mathrm{q}_{2}$ & $172>126$ & 20 & \\
\hline 10.49 & & Ethalfluralin & $\mathrm{Q}$ & $334>232$ & 10 & 20 \\
\hline & & & $\mathrm{q}_{1}$ & $334>186$ & 30 & \\
\hline & & & $\mathrm{q}_{2}$ & $334>300$ & 20 & \\
\hline 10.5 & & Terbumeton desethyl & $Q$ & $198>142$ & 20 & 20 \\
\hline & & & $\mathrm{q}_{1}$ & $198>86$ & 30 & \\
\hline & & & $\mathrm{q}_{2}$ & $198>100$ & 30 & \\
\hline 10.5 & & Atrazine desethyl & $\mathrm{Q}$ & $188>146$ & 20 & 10 \\
\hline & & & $\mathrm{q}_{1}$ & $188>104$ & 30 & \\
\hline & & & $\mathrm{q}_{2}$ & $188>111$ & 10 & \\
\hline 10.65 & & Trifluraline & $\mathrm{Q}$ & $336>232$ & 20 & 30 \\
\hline & & & $\mathrm{q}_{1}$ & $336>186$ & 30 & \\
\hline & & & $\mathrm{q}_{2}$ & $336>202$ & 30 & \\
\hline
\end{tabular}


Table 1 (continued).

\begin{tabular}{|c|c|c|c|c|c|c|}
\hline \multirow{2}{*}{$\frac{\mathrm{t}_{\mathrm{R}}(\min )}{10.7}$} & \multirow{2}{*}{$\frac{\text { Window (min) }}{10.5-11.3}$} & \multirow{2}{*}{$\begin{array}{l}\text { Compounds } \\
\text { Terbutylazine desethyl }\end{array}$} & \multicolumn{2}{|c|}{ SRM Transitions } & \multirow{2}{*}{$\begin{array}{c}\begin{array}{c}\text { Collision } \\
\text { energy (eV) }\end{array} \\
20\end{array}$} & \multirow{2}{*}{$\begin{array}{c}\text { Cone voltage } \\
\text { (V) }\end{array}$} \\
\hline & & & $\mathrm{Q}$ & $202>146$ & & \\
\hline & & & $\mathrm{q}_{1}$ & $202>104$ & 30 & \\
\hline & & & $\mathrm{q}_{2}$ & $202>116$ & 30 & \\
\hline \multirow{3}{*}{10.79} & & Cadusafos & $\mathrm{Q}$ & $271>131$ & 10 & 30 \\
\hline & & & $\mathrm{q}_{1}$ & $271>97$ & 10 & \\
\hline & & & $\mathrm{q}_{2}$ & $271>125$ & 20 & \\
\hline \multirow[t]{3}{*}{10.87} & & Phorate & $\mathrm{Q}$ & $261>97$ & 30 & 20 \\
\hline & & & $\mathrm{q}_{1}$ & $261>171$ & 10 & \\
\hline & & & $\mathrm{q}_{2}$ & $261>199$ & 10 & \\
\hline \multirow[t]{3}{*}{10.97} & & alpha-HCH & $\mathrm{Q}$ & $181>146$ & 20 & 10 \\
\hline & & & $\mathrm{q}_{1}$ & $181>109$ & 30 & \\
\hline & & & $\mathrm{q}_{2}$ & $217>181$ & 10 & \\
\hline \multirow[t]{3}{*}{11.12} & & Hexachlorobenzene & $\mathrm{Q}$ & $282>247$ & 30 & 40 \\
\hline & & & $\mathrm{q}_{1}$ & $282>177$ & 30 & \\
\hline & & & $\mathrm{q}_{2}$ & $282>212$ & 30 & \\
\hline \multirow[t]{3}{*}{11.2} & & Dichloran & $\mathrm{Q}$ & $207>190$ & 20 & 10 \\
\hline & & & $\mathrm{q}_{1}$ & $207>124$ & 30 & \\
\hline & & & $\mathrm{q}_{2}$ & $207>160$ & 20 & \\
\hline \multirow[t]{3}{*}{11.2} & & Dimethoate & $\mathrm{Q}$ & $230>125$ & 20 & 30 \\
\hline & & & $\mathrm{q}_{1}$ & $230>171$ & 10 & \\
\hline & & & $\mathrm{q}_{2}$ & $230>199$ & 10 & \\
\hline \multirow[t]{3}{*}{11.25} & $11-12$ & Ethoxyquin & $\mathrm{Q}$ & $218>174$ & 30 & 30 \\
\hline & & & $\mathrm{q}_{1}$ & $218>160$ & 30 & \\
\hline & & & $\mathrm{q}_{2}$ & $218>202$ & 20 & \\
\hline \multirow[t]{3}{*}{11.27} & & Simazine & $\mathrm{Q}$ & $202>132$ & 20 & 30 \\
\hline & & & $\mathrm{q}_{1}$ & $202>104$ & 30 & \\
\hline & & & $\mathrm{q}_{2}$ & $202>124$ & 20 & \\
\hline \multirow[t]{3}{*}{11.38} & & Atrazine & $\mathrm{Q}$ & $216>174$ & 20 & 10 \\
\hline & & & $\mathrm{q}_{1}$ & $216>104$ & 30 & \\
\hline & & & $\mathrm{q}_{2}$ & $216>132$ & 30 & \\
\hline 11.47 & & beta-HCH & $\mathrm{Q}$ & $181>146$ & 20 & 10 \\
\hline & & & $\mathrm{q}_{1}$ & $181>109$ & 30 & \\
\hline & & & $\mathrm{q}_{2}$ & $217>181$ & 10 & \\
\hline 11.49 & & Terbumeton & $\mathrm{Q}$ & $226>170$ & 20 & 30 \\
\hline & & & $\mathrm{q}_{1}$ & $226>114$ & 30 & \\
\hline & & & $\mathrm{q}_{2}$ & $226>142$ & 30 & \\
\hline 11.6 & $11.5-11.85$ & Dioxathion & $\mathrm{Q}$ & $271>97$ & 30 & 20 \\
\hline & & & $\mathrm{q}_{1}$ & $271>125$ & 10 & \\
\hline & & & $\mathrm{q}_{2}$ & $271>141$ & 20 & \\
\hline 11.6 & & gamma-HCH & $\mathrm{Q}$ & $181>146$ & 20 & 10 \\
\hline & & & $\mathrm{q}_{1}$ & $181>109$ & 30 & \\
\hline & & & $\mathrm{q}_{2}$ & $217>181$ & 10 & \\
\hline 11.66 & & Terbutylazine & $\mathrm{Q}$ & $230>174$ & 20 & 20 \\
\hline & & & $\mathrm{q}_{1}$ & $230>104$ & 30 & \\
\hline & & & $\mathrm{q}_{2}$ & $230>132$ & 30 & \\
\hline 11.67 & & Propetamphos & $\mathrm{Q}$ & $222>138$ & 10 & 20 \\
\hline & & & $\mathrm{q}_{1}$ & $222>110$ & 20 & \\
\hline & & & $\mathrm{q}_{2}$ & $282>138$ & 20 & \\
\hline 11.68 & & Cyanophos & $\mathrm{Q}$ & $244>125$ & 30 & 5 \\
\hline & & & $\mathrm{q}_{1}$ & $244>134$ & 30 & \\
\hline & & & $\mathrm{q}_{2}$ & $244>150$ & 20 & \\
\hline 11.68 & & Terbufos & $\mathrm{Q}$ & $187>97$ & 10 & 5 \\
\hline & & & $\mathrm{q}_{1}$ & $187>131$ & 20 & \\
\hline & & & $\mathrm{q}_{2}$ & $187>159$ & 10 & \\
\hline 11.72 & & Propyzamide & $\mathrm{Q}$ & $256>190$ & 10 & 30 \\
\hline & & & $\mathrm{q}_{1}$ & $256>145$ & 30 & \\
\hline & & & $\mathrm{q}_{2}$ & $256>173$ & 20 & \\
\hline 11.9 & $11.7-12.25$ & Diazinon & Q & $305>169$ & 30 & 40 \\
\hline & & & $\mathrm{q}_{1}$ & $305>153$ & 30 & \\
\hline & & & $\mathrm{q}_{2}$ & $305>249$ & 20 & \\
\hline
\end{tabular}


Table 1 (continued).

\begin{tabular}{|c|c|c|c|c|c|c|}
\hline \multirow{2}{*}{$\frac{\mathrm{t}_{\mathrm{R}}(\mathrm{min})}{12.01}$} & \multirow{2}{*}{ Window (min) } & \multirow{2}{*}{$\begin{array}{l}\text { Compounds } \\
\text { Terbacil }\end{array}$} & \multicolumn{2}{|c|}{ SRM Transitions } & \multirow{2}{*}{$\begin{array}{c}\begin{array}{c}\text { Collision } \\
\text { energy }(\mathrm{eV})\end{array} \\
20\end{array}$} & \multirow{2}{*}{$\begin{array}{c}\begin{array}{c}\text { Cone voltage } \\
\text { (V) }\end{array} \\
20\end{array}$} \\
\hline & & & $\mathrm{Q}$ & $161>144$ & & \\
\hline & & & $\mathrm{q}_{1}$ & $161>118$ & 20 & \\
\hline & & & $\mathrm{q}_{2}$ & $161>143$ & 20 & \\
\hline \multirow{3}{*}{12.05} & & delta-HCH & $Q$ & $181>146$ & 20 & 30 \\
\hline & & & $\mathrm{q}_{1}$ & $181>109$ & 30 & \\
\hline & & & $\mathrm{q}_{2}$ & $217>181$ & 10 & \\
\hline \multirow[t]{3}{*}{12.08} & & Tefluthrin & $\mathrm{Q}$ & $177>127$ & 20 & 5 \\
\hline & & & $\mathrm{q}_{1}$ & $419>177$ & 10 & \\
\hline & & & $\mathrm{q}_{2}$ & $419>325$ & 10 & \\
\hline \multirow[t]{3}{*}{12.14} & & Chlorothalonil & $\mathrm{Q}$ & $265>230$ & 20 & 30 \\
\hline & & & $\mathrm{q}_{1}$ & $265>133$ & 30 & \\
\hline & & & $\mathrm{q}_{2}$ & $265>211$ & 20 & \\
\hline \multirow[t]{3}{*}{12.4} & $12.25-12.7$ & Pirimicarb & $\mathrm{Q}$ & $239>182$ & 20 & 10 \\
\hline & & & $\mathrm{q}_{1}$ & $239>109$ & 30 & \\
\hline & & & $\mathrm{q}_{2}$ & $239>138$ & 30 & \\
\hline \multirow[t]{3}{*}{12.43} & & Endosulfan ether & $Q$ & $341>217$ & 30 & 30 \\
\hline & & & $\mathrm{q}_{1}$ & $341>170$ & 30 & \\
\hline & & & $\mathrm{q}_{2}$ & $341>205$ & 20 & \\
\hline \multirow[t]{3}{*}{12.62} & & Phosphamidon & $\mathrm{Q}$ & $300>127$ & 20 & 40 \\
\hline & & & $\mathrm{q}_{1}$ & $300>174$ & 10 & \\
\hline & & & $\mathrm{q}_{2}$ & $300>227$ & 10 & \\
\hline \multirow[t]{3}{*}{12.62} & & Dichlofenthion & $\mathrm{Q}$ & $315>259$ & 20 & 30 \\
\hline & & & $\mathrm{q}_{1}$ & $315>179$ & 20 & \\
\hline & & & $\mathrm{q}_{2}$ & $315>287$ & 10 & \\
\hline \multirow[t]{3}{*}{12.66} & $12.4-13.15$ & Metribuzin & $\mathrm{Q}$ & $215>187$ & 20 & 40 \\
\hline & & & $\mathrm{q}_{1}$ & $215>145$ & 20 & \\
\hline & & & $\mathrm{q}_{2}$ & $215>171$ & 20 & \\
\hline \multirow[t]{3}{*}{12.79} & & Vinclozolin & $Q$ & $286>242$ & 10 & 5 \\
\hline & & & $\mathrm{q}_{1}$ & $286>164$ & 30 & \\
\hline & & & $\mathrm{q}_{2}$ & $286>172$ & 20 & \\
\hline 12.8 & & Parathion methyl & $\mathrm{q}^{2}$ & $264>232$ & 20 & 20 \\
\hline & & & $\mathrm{q}_{1}$ & $264>125$ & 30 & \\
\hline & & & $\mathrm{q}_{2}$ & $264>155$ & 30 & \\
\hline 12.8 & & Chlorpyrifos methyl & $\mathrm{Q}$ & $322>125$ & 30 & 40 \\
\hline & & & $\mathrm{q}_{1}$ & $322>212$ & 30 & \\
\hline & & & $\mathrm{q}_{2}$ & $322>290$ & 20 & \\
\hline 12.94 & & Alachlor & $Q$ & $238>162$ & 20 & 30 \\
\hline & & & $\mathrm{q}_{1}$ & $238>132$ & 30 & \\
\hline & & & $\mathrm{q}_{2}$ & $270>147$ & 30 & \\
\hline 12.96 & & Heptachlor & $Q$ & $335>264$ & 20 & 40 \\
\hline & & & $\mathrm{q}_{1}$ & $335>230$ & 30 & \\
\hline & & & $\mathrm{q}_{2}$ & $335>299$ & 10 & \\
\hline 13.03 & & Metalaxyl & $\mathrm{Q}$ & $280>220$ & 10 & 30 \\
\hline & & & $\mathrm{q}_{1}$ & $280>160$ & 20 & \\
\hline & & & $\mathrm{q}_{2}$ & $280>192$ & 10 & \\
\hline 13.1 & $12.8-13.6$ & Methiocarb sulfone & $\mathrm{Q}$ & $201>122$ & 10 & 20 \\
\hline & & & $\mathrm{q}_{1}$ & $201>91$ & 30 & \\
\hline & & & $\mathrm{q}_{2}$ & $201>107$ & 30 & \\
\hline 13.15 & & Demeton-s-methylsulfone & $\mathrm{Q}$ & $263>125$ & 20 & 30 \\
\hline & & & $\mathrm{q}_{1}$ & $263>169$ & 10 & \\
\hline & & & $\mathrm{q}_{2}$ & $263>231$ & 10 & \\
\hline 13.25 & & Terbutryn & $\mathrm{Q}$ & $242>186$ & 20 & 40 \\
\hline & & & $\mathrm{q}_{1}$ & $242>116$ & 30 & \\
\hline & & & $\mathrm{q}_{2}$ & $242>138$ & 30 & \\
\hline 13.3 & & Methiocarb & $\mathrm{Q}$ & $226>169$ & 10 & 30 \\
\hline & & & $\mathrm{q}_{1}$ & $226>121$ & 20 & \\
\hline & & & $\mathrm{q}_{2}$ & $226>122$ & 30 & \\
\hline 13.3 & & Fenitrothion & $\mathrm{Q}$ & $278>125$ & 30 & 40 \\
\hline & & & $\mathrm{q}_{1}$ & $278>169$ & 30 & \\
\hline & & & $\mathrm{q}_{2}$ & $278>200$ & 20 & \\
\hline
\end{tabular}


Table 1 (continued).

\begin{tabular}{|c|c|c|c|c|c|c|}
\hline \multirow{2}{*}{$\frac{\mathrm{t}_{\mathrm{R}}(\min )}{13.34}$} & \multirow[t]{2}{*}{ Window (min) } & \multirow{2}{*}{$\begin{array}{l}\text { Compounds } \\
\text { Pirimiphos methyl }\end{array}$} & \multicolumn{2}{|c|}{ SRM Transitions } & \multirow{2}{*}{$\begin{array}{c}\begin{array}{c}\text { Collision } \\
\text { energy }(\mathrm{eV})\end{array} \\
30\end{array}$} & \multirow{2}{*}{$\begin{array}{c}\begin{array}{c}\text { Cone voltage } \\
\text { (V) }\end{array} \\
5\end{array}$} \\
\hline & & & $\mathrm{Q}$ & $306>125$ & & \\
\hline & & & $\mathrm{q}_{1}$ & $306>109$ & 30 & \\
\hline & & & $\mathrm{q}_{2}$ & $306>164$ & 30 & \\
\hline \multirow{3}{*}{13.5} & & Malathion & $Q$ & $331>125$ & 30 & 20 \\
\hline & & & $\mathrm{q}_{1}$ & $331>117$ & 20 & \\
\hline & & & $\mathrm{q}_{2}$ & $331>211$ & 10 & \\
\hline \multirow[t]{3}{*}{13.61} & $13.3-13.85$ & Aldrin & $\mathrm{Q}$ & $363>159$ & 20 & 30 \\
\hline & & & $\mathrm{q}_{1}$ & $363>215$ & 20 & \\
\hline & & & $\mathrm{q}_{2}$ & $363>327$ & 10 & \\
\hline \multirow[t]{3}{*}{13.63} & & Metolachlor & $\mathrm{Q}$ & $284>252$ & 20 & 20 \\
\hline & & & $\mathrm{q}_{1}$ & $284>134$ & 30 & \\
\hline & & & $\mathrm{q}_{2}$ & $284>176$ & 30 & \\
\hline \multirow[t]{3}{*}{13.66} & & Fenthion & $\mathrm{Q}$ & $279>247$ & 10 & 20 \\
\hline & & & $\mathrm{q}_{1}$ & $279>105$ & 20 & \\
\hline & & & $\mathrm{q}_{2}$ & $279>169$ & 30 & \\
\hline \multirow[t]{3}{*}{13.68} & & Cyanazine & $\mathrm{Q}$ & $241>214$ & 20 & 30 \\
\hline & & & $\mathrm{q}_{1}$ & $241>132$ & 30 & \\
\hline & & & $\mathrm{q}_{2}$ & $241>205$ & 20 & \\
\hline \multirow[t]{3}{*}{13.71} & & Chlorpyrifos & $\mathrm{Q}$ & $350>198$ & 20 & 20 \\
\hline & & & $\mathrm{q}_{1}$ & $350>294$ & 10 & \\
\hline & & & $\mathrm{q}_{2}$ & $350>322$ & 10 & \\
\hline \multirow[t]{3}{*}{13.72} & & Parathion-ethyl & $\mathrm{Q}$ & $292>236$ & 20 & 20 \\
\hline & & & $\mathrm{q}_{1}$ & $292>110$ & 30 & \\
\hline & & & $\mathrm{q}_{2}$ & $292>123$ & 30 & \\
\hline \multirow[t]{3}{*}{13.76} & & Triadimefon & $\mathrm{Q}$ & $294>197$ & 10 & 40 \\
\hline & & & $\mathrm{q}_{1}$ & $294>129$ & 20 & \\
\hline & & & $\mathrm{q}_{2}$ & $294>141$ & 20 & \\
\hline \multirow[t]{3}{*}{13.76} & & 4,4'-Dichloronbenzophenone & $\mathrm{Q}$ & $251>139$ & 20 & 20 \\
\hline & & & $\mathrm{q}_{1}$ & $251>111$ & 30 & \\
\hline & & & $\mathrm{q}_{2}$ & $251>129$ & 30 & \\
\hline 14.04 & $13.85-14.4$ & Bromophos methyl & $\mathrm{Q}$ & $365>125$ & 20 & 30 \\
\hline & & & $\mathrm{q}_{1}$ & $365>211$ & 30 & \\
\hline & & & $\mathrm{q}_{2}$ & $365>239$ & 30 & \\
\hline 14.15 & & Isodrin & $\mathrm{Q}$ & $363>159$ & 20 & 30 \\
\hline & & & $\mathrm{q}_{1}$ & $363>215$ & 20 & \\
\hline & & & $\mathrm{q}_{2}$ & $363>327$ & 10 & \\
\hline 14.16 & & Cyprodinil & $\mathrm{Q}$ & $226>118$ & 30 & 40 \\
\hline & & & $\mathrm{q}_{1}$ & $226>133$ & 30 & \\
\hline & & & $\mathrm{q}_{2}$ & $226>210$ & 30 & \\
\hline 14.3 & & Pendimethalin & $\mathrm{Q}$ & $282>212$ & 10 & 20 \\
\hline & & & $\mathrm{q}_{1}$ & $264>147$ & 30 & \\
\hline & & & $\mathrm{q}_{2}$ & $264>201$ & 20 & \\
\hline 14.35 & $14.1-14.6$ & Heptachlor epoxide B & $\mathrm{Q}$ & $351>251$ & 30 & 20 \\
\hline & & & $\mathrm{q}_{1}$ & $351>217$ & 20 & \\
\hline & & & $\mathrm{q}_{2}$ & $351>287$ & 10 & \\
\hline 14.37 & & Oxychlordane & $\mathrm{Q}$ & $421>151$ & 20 & 10 \\
\hline & & & $\mathrm{q}_{1}$ & $42 I>115$ & 20 & \\
\hline & & & $\mathrm{q}_{2}$ & $421>285$ & 30 & \\
\hline 14.41 & & Tolyfluanid & $\mathrm{Q}$ & $238>137$ & 20 & 5 \\
\hline & & & $\mathrm{q}_{1}$ & $238>110$ & 30 & \\
\hline & & & $\mathrm{q}_{2}$ & $238>122$ & 30 & \\
\hline 14.43 & & Heptachlor epoxide A & $\mathrm{Q}$ & $351>251$ & 30 & 20 \\
\hline & & & $\mathrm{q}_{1}$ & $351>217$ & 20 & \\
\hline & & & $\mathrm{q}_{2}$ & $351>287$ & 10 & \\
\hline 14.46 & & Chlorfenvinphos & $\mathrm{Q}$ & $359>170$ & 30 & 30 \\
\hline & & & $\mathrm{q}_{1}$ & $359>99$ & 10 & \\
\hline & & & $\mathrm{q}_{2}$ & $359>205$ & 20 & \\
\hline 14.47 & & Fipronil & $Q$ & $437>368$ & 20 & 30 \\
\hline & & & $\mathrm{q}_{1}$ & $437>255$ & 30 & \\
\hline & & & $\mathrm{q}_{2}$ & $437>315$ & 30 & \\
\hline
\end{tabular}


Table 1 (continued).

\begin{tabular}{|c|c|c|c|c|c|c|}
\hline \multirow{2}{*}{$\frac{\mathrm{t}_{\mathrm{R}}(\min )}{14.5}$} & \multirow{2}{*}{$\frac{\text { Window (min) }}{14.2-14.8}$} & \multirow{2}{*}{$\begin{array}{l}\text { Compounds } \\
\text { Captan }\end{array}$} & \multicolumn{2}{|c|}{ SRM Transitions } & \multirow{2}{*}{$\begin{array}{c}\begin{array}{c}\text { Collision } \\
\text { energy }(\mathrm{eV})\end{array} \\
10\end{array}$} & \multirow{2}{*}{$\begin{array}{c}\begin{array}{c}\text { Cone voltage } \\
\text { (V) }\end{array} \\
30\end{array}$} \\
\hline & & & $\mathrm{Q}$ & $264>236$ & & \\
\hline & & & $\mathrm{q}_{1}$ & $264>156$ & 30 & \\
\hline & & & $\mathrm{q}_{2}$ & $264>180$ & 10 & \\
\hline \multirow{3}{*}{14.52} & & Quinalphos & $\mathrm{Q}$ & $299>163$ & 20 & 30 \\
\hline & & & $\mathrm{q}_{1}$ & $299>147$ & 30 & \\
\hline & & & $\mathrm{q}_{2}$ & $299>271$ & 10 & \\
\hline \multirow[t]{3}{*}{14.6} & & Folpet & $\mathrm{Q}$ & $260>130$ & 20 & 5 \\
\hline & & & $\mathrm{q}_{1}$ & $260>102$ & 30 & \\
\hline & & & $\mathrm{q}_{2}$ & $260>232$ & 10 & \\
\hline \multirow[t]{3}{*}{14.63} & & Procymidone & $\mathrm{Q}$ & $284>256$ & 20 & 30 \\
\hline & & & $\mathrm{q}_{1}$ & $284>186$ & 30 & \\
\hline & & & $\mathrm{q}_{2}$ & $284>228$ & 20 & \\
\hline \multirow[t]{3}{*}{14.67} & & Triflumizole & $\mathrm{Q}$ & $346>278$ & 10 & 10 \\
\hline & & & $\mathrm{q}_{1}$ & $346>206$ & 20 & \\
\hline & & & $\mathrm{q}_{2}$ & $346>266$ & 20 & \\
\hline \multirow[t]{3}{*}{14.79} & $14.4-15.3$ & Chinomethionate & $\mathrm{Q}$ & $235>175$ & 20 & 30 \\
\hline & & & $\mathrm{q}_{1}$ & $235>104$ & 30 & \\
\hline & & & $\mathrm{q}_{2}$ & $235>121$ & 30 & \\
\hline \multirow[t]{3}{*}{14.8} & & Methidathion & $\mathrm{Q}$ & $303>145$ & 10 & 10 \\
\hline & & & $\mathrm{q}_{1}$ & $303>125$ & 20 & \\
\hline & & & $\mathrm{q}_{2}$ & $303>257$ & 10 & \\
\hline \multirow[t]{3}{*}{14.8} & & trans-Chlordane & $\mathrm{Q}$ & $371>264$ & 30 & 10 \\
\hline & & & $\mathrm{q}_{1}$ & $371>299$ & 20 & \\
\hline & & & $\mathrm{q}_{2}$ & $371>335$ & 20 & \\
\hline \multirow[t]{3}{*}{14.82} & & Bromophos ethyl & $\mathrm{Q}$ & $393>337$ & 20 & 10 \\
\hline & & & $\mathrm{q}_{1}$ & $393>162$ & 30 & \\
\hline & & & $\mathrm{q}_{2}$ & $393>365$ & 10 & \\
\hline \multirow[t]{3}{*}{15.01} & & Endosulfan I & $Q$ & $405>323$ & 10 & 5 \\
\hline & & & $\mathrm{q}_{1}$ & $405>217$ & 30 & \\
\hline & & & $\mathrm{q}_{2}$ & $405>251$ & 20 & \\
\hline 15.14 & & Fenamiphos & $\mathrm{Q}$ & $304>217$ & 20 & 40 \\
\hline & & & $\mathrm{q}_{1}$ & $304>202$ & 30 & \\
\hline & & & $\mathrm{q}_{2}$ & $304>234$ & 20 & \\
\hline 15.17 & $15-15.8$ & Chlorfenson & $\mathrm{Q}$ & $303>159$ & 10 & 5 \\
\hline & & & $\mathrm{q}_{1}$ & $303>111$ & 10 & \\
\hline & & & $\mathrm{q}_{2}$ & $303>128$ & 30 & \\
\hline 15.32 & & Imazalil & $\mathrm{Q}$ & $297>159$ & 20 & 10 \\
\hline & & & $\mathrm{q}_{1}$ & $297>109$ & 20 & \\
\hline & & & $\mathrm{q}_{2}$ & $297>176$ & 20 & \\
\hline 15.36 & & Fludioxonil & $\mathrm{Q}$ & $248>127$ & 30 & 30 \\
\hline & & & $\mathrm{q}_{1}$ & $248>154$ & 20 & \\
\hline & & & $\mathrm{q}_{2}$ & $248>182$ & 20 & \\
\hline 15.43 & & $p, p^{\prime}-\mathrm{DDE}$ & $\mathrm{Q}$ & $316>246$ & 30 & 20 \\
\hline & & & $\mathrm{q}_{1}$ & $316>210$ & 30 & \\
\hline & & & $\mathrm{q}_{2}$ & $316>281$ & 20 & \\
\hline 15.49 & & Dieldrin & $\mathrm{Q}$ & $379>325$ & 10 & 20 \\
\hline & & & $\mathrm{q}_{1}$ & $379>254$ & 30 & \\
\hline & & & $\mathrm{q}_{2}$ & $379>261$ & 20 & \\
\hline 15.57 & & Oxyfluorfen & $\mathrm{Q}$ & $362>316$ & 10 & 30 \\
\hline & & & $\mathrm{q}_{1}$ & $362>237$ & 20 & \\
\hline & & & $\mathrm{q}_{2}$ & $362>334$ & 10 & \\
\hline 15.61 & & Buprofezin & $\mathrm{Q}$ & $306>106$ & 20 & 30 \\
\hline & & & $\mathrm{q}_{1}$ & $306>203$ & 10 & \\
\hline & & & $\mathrm{q}_{2}$ & $306>250$ & 10 & \\
\hline 15.9 & $15.6-16.4$ & Endrin & $\mathrm{Q}$ & $379>343$ & 10 & 30 \\
\hline & & & $\mathrm{q}_{1}$ & $379>243$ & 20 & \\
\hline & & & $\mathrm{q}_{2}$ & $379>244$ & 20 & \\
\hline 16.05 & & Endosulfan II & Q & $405>323$ & 10 & 30 \\
\hline & & & $\mathrm{q}_{1}$ & $405>217$ & 30 & \\
\hline & & & $\mathrm{q}_{2}$ & $405>251$ & 20 & \\
\hline
\end{tabular}


Table 1 (continued).

\begin{tabular}{|c|c|c|c|c|c|c|}
\hline \multirow{2}{*}{$\frac{t_{R}(\min )}{16.18}$} & \multirow[t]{2}{*}{ Window (min) } & \multirow{2}{*}{$\begin{array}{l}\text { Compounds } \\
p, p^{\prime} \text {-DDD }\end{array}$} & \multicolumn{2}{|c|}{ SRM Transitions } & \multirow{2}{*}{$\begin{array}{c}\begin{array}{c}\text { Collision } \\
\text { energy }(\mathrm{eV})\end{array} \\
20\end{array}$} & \multirow{2}{*}{$\begin{array}{c}\text { Cone voltage } \\
\text { (V) }\end{array}$} \\
\hline & & & $\mathrm{Q}$ & $235>165$ & & \\
\hline & & & $\mathrm{q}_{1}$ & $235>99$ & 30 & \\
\hline & & & $\mathrm{q}_{2}$ & $235>199$ & 20 & \\
\hline \multirow{3}{*}{16.25} & & Oxadixyl & $\mathrm{Q}$ & $279>219$ & 10 & 5 \\
\hline & & & $\mathrm{q}_{1}$ & $279>117$ & 30 & \\
\hline & & & $q_{2}$ & $279>132$ & 20 & \\
\hline \multirow[t]{3}{*}{16.27} & & Ethion & $\mathrm{Q}$ & $385>143$ & 30 & 5 \\
\hline & & & $\mathrm{q}_{1}$ & $385>97$ & 10 & \\
\hline & & & $\mathrm{q}_{2}$ & $385>125$ & 20 & \\
\hline \multirow[t]{3}{*}{16.47} & $16.3-16.8$ & Sulprofos & $\mathrm{Q}$ & $323>139$ & 20 & 10 \\
\hline & & & $\mathrm{q}_{1}$ & $323>155$ & 30 & \\
\hline & & & $\mathrm{q}_{2}$ & $323>219$ & 10 & \\
\hline \multirow[t]{3}{*}{16.62} & & Famphur & $\mathrm{Q}$ & $326>217$ & 20 & 30 \\
\hline & & & $\mathrm{q}_{1}$ & $326>125$ & 20 & \\
\hline & & & $\mathrm{q}_{2}$ & $326>152$ & 30 & \\
\hline \multirow[t]{3}{*}{16.65} & & Carbofenothion & $\mathrm{Q}$ & $343>157$ & 20 & 5 \\
\hline & & & $\mathrm{q}_{1}$ & $343>97$ & 30 & \\
\hline & & & $\mathrm{q}_{2}$ & $343>121$ & 30 & \\
\hline \multirow[t]{3}{*}{16.67} & & Carfentrazone ethyl & $\mathrm{Q}$ & $412>346$ & 20 & 10 \\
\hline & & & $\mathrm{q}_{1}$ & $412>366$ & 20 & \\
\hline & & & $\mathrm{q}_{2}$ & $412>384$ & 10 & \\
\hline \multirow[t]{3}{*}{16.78} & $16.5-17$ & Propiconazole & $\mathrm{Q}$ & $342>159$ & 20 & 30 \\
\hline & & & $\mathrm{q}_{1}$ & $342>187$ & 20 & \\
\hline & & & $\mathrm{q}_{2}$ & $342>256$ & 10 & \\
\hline \multirow[t]{3}{*}{16.8} & & Endosulfan sulfate & $\mathrm{Q}$ & $323>217$ & 30 & 10 \\
\hline & & & $\mathrm{q}_{1}$ & $323>251$ & 20 & \\
\hline & & & $\mathrm{q}_{2}$ & $323>287$ & 10 & \\
\hline \multirow[t]{3}{*}{16.84} & & Fenhexamid & $\mathrm{Q}$ & $302>143$ & 30 & 30 \\
\hline & & & $\mathrm{q}_{1}$ & $302>142$ & 30 & \\
\hline & & & $\mathrm{q}_{2}$ & $302>178$ & 20 & \\
\hline 16.85 & & $p, p^{\prime}-\mathrm{DDT}$ & $\mathrm{Q}$ & $235>165$ & 20 & 5 \\
\hline & & & $\mathrm{q}_{1}$ & $235>99$ & 30 & \\
\hline & & & $\mathrm{q}_{2}$ & $235>199$ & 20 & \\
\hline 17.17 & $16.9-17.9$ & Diflufenican & $\mathrm{Q}$ & $395>266$ & 20 & 10 \\
\hline & & & $\mathrm{q}_{1}$ & $395>238$ & 30 & \\
\hline & & & $\mathrm{q}_{2}$ & $395>246$ & 30 & \\
\hline 17.23 & & Captafol & $\mathrm{Q}$ & $348>312$ & 10 & 10 \\
\hline & & & $\mathrm{q}_{1}$ & $348>117$ & 30 & \\
\hline & & & $\mathrm{q}_{2}$ & $348>161$ & 20 & \\
\hline 17.27 & & Resmethrin & $\mathrm{Q}$ & $339>171$ & 10 & 30 \\
\hline & & & $\mathrm{q}_{1}$ & $339>143$ & 20 & \\
\hline & & & $\mathrm{q}_{2}$ & $339>293$ & 10 & \\
\hline 17.54 & & Iprodione & $\mathrm{Q}$ & $330>245$ & 10 & 30 \\
\hline & & & $\mathrm{q}_{1}$ & $330>174$ & 30 & \\
\hline & & & $\mathrm{q}_{2}$ & $330>288$ & 10 & \\
\hline 17.71 & & Fenoxycarb & $\mathrm{Q}$ & $302>256$ & 10 & 40 \\
\hline & & & $\mathrm{q}_{1}$ & $302>183$ & 20 & \\
\hline & & & $\mathrm{q}_{2}$ & $302>213$ & 20 & \\
\hline 17.71 & & Phosmet & $\mathrm{Q}$ & $318>160$ & 10 & 20 \\
\hline & & & $\mathrm{q}_{1}$ & $160>133$ & 20 & \\
\hline & & & $\mathrm{q}_{2}$ & $318>133$ & 30 & \\
\hline 17.77 & & Bifenthrin & $\mathrm{Q}$ & $181>165$ & 20 & 10 \\
\hline & & & $\mathrm{q}_{1}$ & $181>115$ & 30 & \\
\hline & & & $\mathrm{q}_{2}$ & $181>166$ & 30 & \\
\hline 17.86 & $17.6-18.7$ & Methoxychlor & $\mathrm{Q}$ & $345>213$ & 20 & 10 \\
\hline & & & $\mathrm{q}_{1}$ & $227>141$ & 30 & \\
\hline & & & $\mathrm{q}_{2}$ & $227>169$ & 30 & \\
\hline 18.22 & & Tetradifon & $\mathrm{Q}$ & $355>195$ & 20 & 30 \\
\hline & & & $\mathrm{q}_{1}$ & $355>133$ & 30 & \\
\hline & & & $\mathrm{q}_{2}$ & $355>167$ & 20 & \\
\hline
\end{tabular}


Table 1 (continued).

\begin{tabular}{|c|c|c|c|c|c|c|}
\hline \multirow{2}{*}{$\frac{\mathrm{t}_{\mathrm{R}}(\min )}{18.4}$} & \multirow[t]{2}{*}{ Window (min) } & \multirow{2}{*}{$\begin{array}{l}\text { Compounds } \\
\text { Azinphos methyl }\end{array}$} & \multicolumn{2}{|c|}{ SRM Transitions } & \multirow{2}{*}{$\begin{array}{c}\begin{array}{c}\text { Collision } \\
\text { energy }(\mathrm{eV})\end{array} \\
20\end{array}$} & \multirow{2}{*}{$\begin{array}{c}\begin{array}{c}\text { Cone voltage } \\
\text { (V) }\end{array} \\
20\end{array}$} \\
\hline & & & $\mathrm{Q}$ & $261>125$ & & \\
\hline & & & $\mathrm{q}_{1}$ & $261>167$ & 10 & \\
\hline & & & $\mathrm{q}_{2}$ & $261>183$ & 10 & \\
\hline \multirow[t]{3}{*}{18.44} & & Leptophos & $\mathrm{Q}$ & $411>171$ & 20 & 40 \\
\hline & & & $\mathrm{q}_{1}$ & $411>139$ & 30 & \\
\hline & & & $\mathrm{q}_{2}$ & $411>379$ & 20 & \\
\hline \multirow[t]{3}{*}{18.45} & & Pyriproxifen & $\mathrm{Q}$ & $322>185$ & 20 & 10 \\
\hline & & & $\mathrm{q}_{1}$ & $322>129$ & 30 & \\
\hline & & & $\mathrm{q}_{2}$ & $322>227$ & 10 & \\
\hline \multirow[t]{3}{*}{18.55} & & lambda-Cyhalothrin & $\mathrm{Q}$ & $450>225$ & 10 & 10 \\
\hline & & & $\mathrm{q}_{1}$ & $450>141$ & 20 & \\
\hline & & & $\mathrm{q}_{2}$ & $450>157$ & 30 & \\
\hline \multirow[t]{3}{*}{18.64} & $18.5-19.8$ & Mirex & Q & $270>235$ & 20 & 10 \\
\hline & & & $\mathrm{q}_{1}$ & $270>117$ & 30 & \\
\hline & & & $\mathrm{q}_{2}$ & $270>141$ & 30 & \\
\hline \multirow[t]{3}{*}{18.9} & & Acrinathrin & Q & $428>401$ & 20 & 10 \\
\hline & & & $\mathrm{q}_{1}$ & $428>205$ & 30 & \\
\hline & & & $\mathrm{q}_{2}$ & $428>260$ & 20 & \\
\hline \multirow[t]{3}{*}{18.88} & & Fenarimol & $\mathrm{Q}$ & $331>268$ & 20 & 40 \\
\hline & & & $\mathrm{q}_{1}$ & $331>139$ & 30 & \\
\hline & & & $\mathrm{q}_{2}$ & $331>259$ & 20 & \\
\hline \multirow[t]{3}{*}{19.01} & & Azinphos ethyl & $\mathrm{Q}$ & $289>137$ & 20 & 20 \\
\hline & & & $\mathrm{q}_{1}$ & $289>233$ & 10 & \\
\hline & & & $\mathrm{q}_{2}$ & $289>261$ & 10 & \\
\hline \multirow[t]{3}{*}{19.43} & & Permethrin & $\mathrm{Q}$ & $355>319$ & 10 & 10 \\
\hline & & & $\mathrm{q}_{1}$ & $391>183$ & 30 & \\
\hline & & & $\mathrm{q}_{2}$ & $391>355$ & 10 & \\
\hline \multirow[t]{3}{*}{19.66} & & Coumaphos & $\mathrm{Q}$ & $363>227$ & 30 & 30 \\
\hline & & & $\mathrm{q}_{1}$ & $363>211$ & 30 & \\
\hline & & & $\mathrm{q}_{2}$ & $363>307$ & 20 & \\
\hline \multirow[t]{3}{*}{20.09} & $19.7-20.35$ & Cyfluthrin & $\mathrm{Q}$ & $434>191$ & 10 & 10 \\
\hline & & & $\mathrm{q}_{1}$ & $434>91$ & 30 & \\
\hline & & & $\mathrm{q}_{2}$ & $434>127$ & 30 & \\
\hline \multirow[t]{3}{*}{20.4} & $20.1-20.85$ & Cypermethrin & $\mathrm{Q}$ & $416>191$ & 10 & 20 \\
\hline & & & $\mathrm{q}_{1}$ & $416>91$ & 30 & \\
\hline & & & $\mathrm{q}_{2}$ & $416>127$ & 30 & \\
\hline 20.51 & & Flucythrinate & $\mathrm{Q}$ & $412>219$ & 30 & 5 \\
\hline & & & $\mathrm{q}_{1}$ & $412>220$ & 30 & \\
\hline & & & $\mathrm{q}_{2}$ & $412>236$ & 30 & \\
\hline 20.59 & & Etofenprox & $\mathrm{Q}$ & $359>183$ & 20 & 10 \\
\hline & & & $\mathrm{q}_{1}$ & $359>161$ & 20 & \\
\hline & & & $\mathrm{q}_{2}$ & $359>289$ & 20 & \\
\hline 21.21 & $20.85-21.6$ & Fenvalerate & $\mathrm{Q}$ & $419>225$ & 10 & 10 \\
\hline & & & $\mathrm{q}_{1}$ & $420>125$ & 10 & \\
\hline & & & $\mathrm{q}_{2}$ & $420>226$ & 10 & \\
\hline 21.38 & & tau-Fluvalinate & $Q^{2}$ & $503>181$ & 20 & 30 \\
\hline & & & $\mathrm{q}_{1}$ & $503>208$ & 30 & \\
\hline & & & $\mathrm{q}_{2}$ & $503>250$ & 20 & \\
\hline 21.4 & & Esfenvalerate & $Q$ & $167>125$ & 10 & 5 \\
\hline & & & $\mathrm{q}_{1}$ & $167>99$ & 30 & \\
\hline & & & $\mathrm{q}_{2}$ & $167>139$ & 10 & \\
\hline 21.94 & $21.7-22.5$ & Deltamethrin & $\mathrm{Q}$ & $504>279$ & 10 & 5 \\
\hline & & & $\mathrm{q}_{1}$ & $504>171$ & 20 & \\
\hline & & & $\mathrm{q}_{2}$ & $504>200$ & 30 & \\
\hline 22.24 & & Azoxystrobin & Q & $404>372$ & 10 & 20 \\
\hline & & & $q_{1}$ & $404>329$ & 30 & \\
\hline & & & $\mathrm{q}_{2}$ & $404>344$ & 20 & \\
\hline
\end{tabular}

Precursors corresponding to $\mathrm{M}^{+} \cdot$ or $[\mathrm{M}+\mathrm{H}]^{+}$are shown in italic. 


\subsection{Sample treatment optimization}

With the QuEChERS sample preparation procedure, the final extract obtained is acetonitrile. The direct injection of the acetonitrile extract was considered less favorable. A (partial) solvent venting using a programmable temperature vaporizer injector could not be done with the GC system used, therefore a solvent exchange step was applied. Initially, in order to avoid evaporation until dryness, $1 \mathrm{~mL}$ of toluene was added to the $500 \quad \mathrm{~L}$ of the acetonitrile extract; evaporation until 300 L using nitrogen stream was performed and then adjusted to $500 \mathrm{~L}$ with toluene. In this way, no losses during the evaporation process were observed. However, the injection of the toluene extracts resulted in a dramatic loss of repeatability. Therefore, a solvent exchange into hexane was tested. In this case evaporation until dryness was unavoidable and the evaporation conditions had to be carefully optimized in order to avoid analyte losses. An evaporation system operating under vacuum was used, which allows a more controlled evaporation and at lower temperature compared with evaporation under nitrogen stream (miVac Modulator Concentrator, provided by Fisher Scientific S.A.S., Illkirch, France). The evaporation was carried out at 30 ${ }^{\circ} \mathrm{C}$ during approximately 30 minutes. However, no satisfactory results were obtained since some notable losses were observed in some analytes with low interday reproducibility.

Then, with the high sensitivity achieved in this GC-(APCI) MS/MS system in mind, the possibility of the direct dilution of the extract with hexane was considered. Standards in acetonitrile at $10 \mathrm{ng} / \mathrm{mL}$ were diluted with hexane (1/10), adding $20 \%$ of acetone to make the solution miscible. It is noteworthy that, in a multi-residue method that includes a large variety of compounds as in this work, the response of the most sensitive compounds are 1000 times higher than those ones with lower sensitivity. Consequently, dilution experiment led to a loss of some analytes that did not show enough sensitivity to be detected. A dilution of $1 / 5$ with hexane (with $20 \%$ of acetone) was also tested but no considerable improvements with respect to the dilution $1 / 10$ were observed for the less sensitive compounds, so this $1 / 10$ dilution (with $20 \%$ of acetone) was selected for further experiments.

Then, experiments were performed by diluting acetonitrile sample extracts fortified at $10 \mathrm{ng} / \mathrm{mL}$ (dilution $1 / 10$ with hexane) and it revealed a significant improvement in peak shapes and sensitivity. In presence of matrix, a higher amount of acetone had to be added 
(30\%) in order to keep the solution miscible. In conclusion, 50

L of acetonitrile extract was mixed with $150 \quad \mathrm{~L}$ of acetone and $300 \quad \mathrm{~L}$ of hexane.

\subsection{Matrix effect}

Matrix effects for all matrices were checked comparing responses of standards in the mixture acetonitrile, hexane and acetone (in the proportions described above), at 10 $\mathrm{ng} / \mathrm{mL}$, with the response of matrix-matched standards (prepared as described in the section "Sample treatment"), at the same concentration. An enhancement of the signal was observed for most compounds except in a few cases such as pyrethroids where a slight suppression occurred, which was in agreement with earlier observations [22]. Matrix effects observed under GC-(APCI) MS are the result of that occurring in the GC inlet (normally enhancement) and in the APCI source (normally suppression). The signal enhancement observed for most compounds can be attributed to that occurring in the GC liner. The matrix shields active sites in the liner and column, which reduces interaction of the analytes on these sites, and leads to enhanced analyte peaks. This effect is most pronounced for polar analytes (typically those with strong hydrogen bonding potential) [25]. Looking at those compounds for which this enhancement is not expected (e.g. hexachlorobenzene, HCHs, etc.), no suppression coming from the APCI source is observed. Thus, it can be concluded that matrix effect observed in GC-(APCI) MS system are mainly arising from the GC inlet and to a lesser extend to suppression from APCI source. For optimum peak shape and sensitivity, as in any GC-based pesticide residue analysis, matrix-matched calibration curves were necessary to perform accurate quantitative analysis.

\subsection{Validation results}

Validation of the method was performed in terms of trueness (recovery) and precision, LODs and LOQs, and selectivity. These parameters were evaluated in three types of matrices, orange, tomato and carrot.

Linearity was studied in the range $0.1-100 \mathrm{ng} / \mathrm{mL}$ using pure solvent standard solutions and adjusted to quadratic curves. Each concentration level was injected in triplicate. The regression coefficients were higher than 0.99 for all compounds over the whole range tested. As mentioned above, matrix-matched calibration was used for quantification purposes. In this case, in order to quantify properly, shorter ranges were 16 
selected depending on the concentration level to be quantified. In this way, residuals were better and lower than $30 \%$.

Trueness and precision were evaluated by means of recovery experiments $(n=6)$ at two concentration levels ( 0.01 and $0.1 \mathrm{mg} / \mathrm{kg}$ ) for each sample matrix. As can be observed in Fig. 1, the histograms show that most compounds presented satisfactory recoveries ranging between $70 \%$ and $120 \%$ for all the sample matrices at the two fortification levels, most of them between $70 \%$ and $110 \%$ (values are presented in Table 2). Thus, an LOQ of $0.01 \mathrm{mg} / \mathrm{kg}$ was demonstrated for most compounds. For the remaining compounds, acceptable results were obtained at $0.1 \mathrm{mg} / \mathrm{kg}$ (e.g. carbaryl in orange and carfentrazoneethyl in carrot). For a limited number of compounds including molinate, propoxur and imazalil, the method was not suitable for the sample matrices and levels tested. Other compounds referred as problematic [26] and [27] as tolyfluanid, chlorothalonil and methiocarb sulfone, did not present satisfactory results in some matrices. RSDs lower than $10 \%$ were obtained for most analytes at both fortification levels, and even lower than $5 \%$, as can be observed in Fig. 2. 


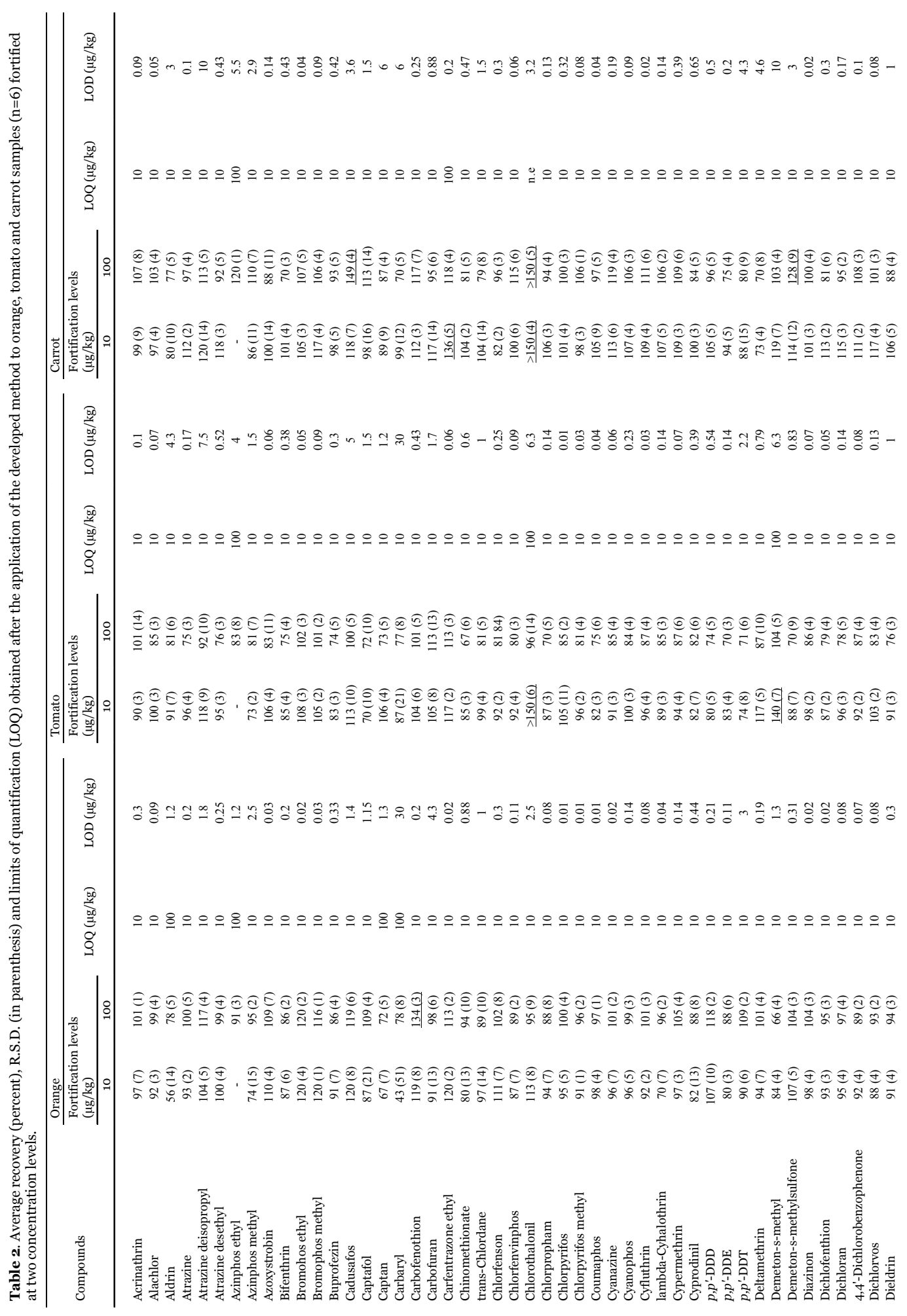




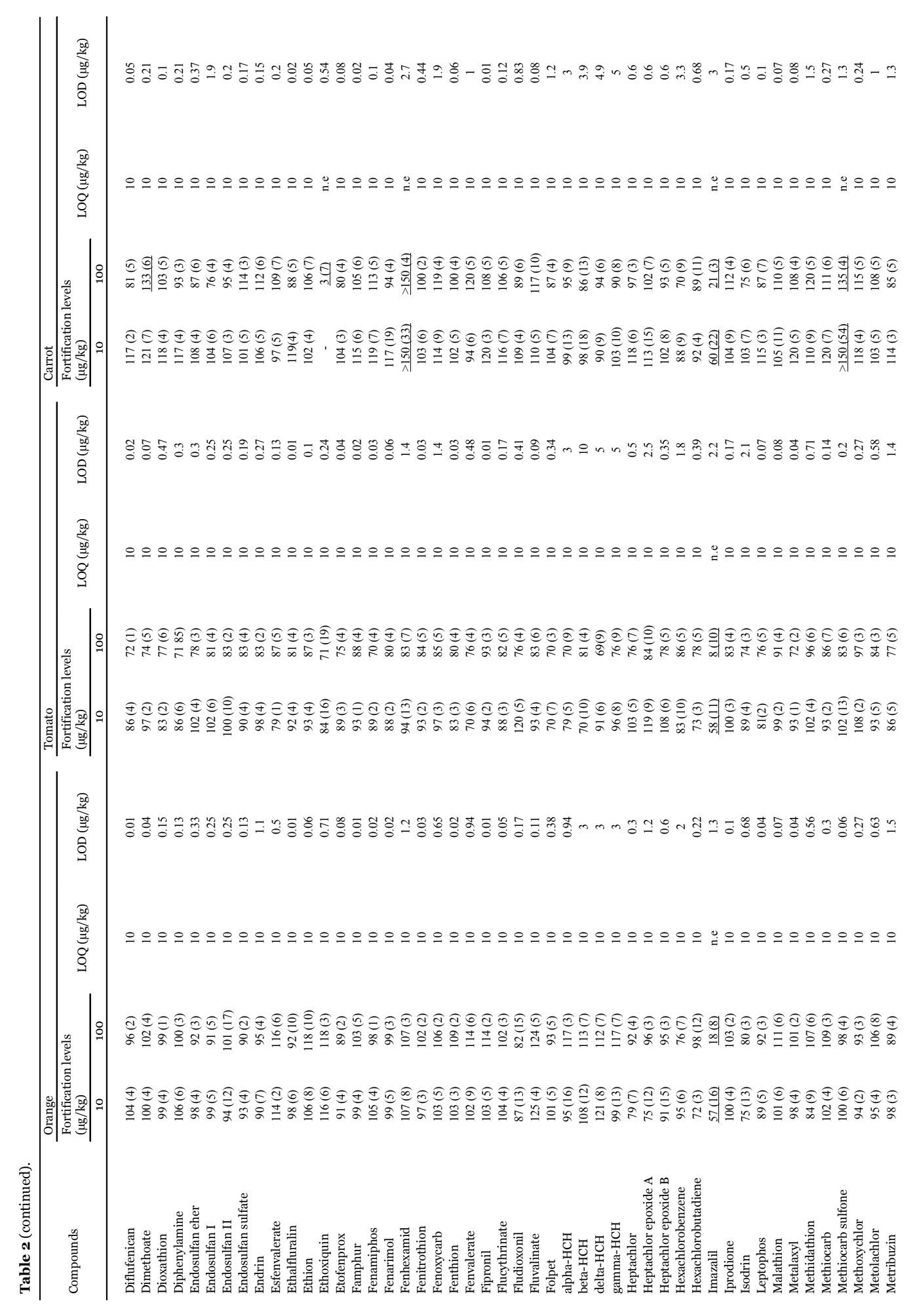




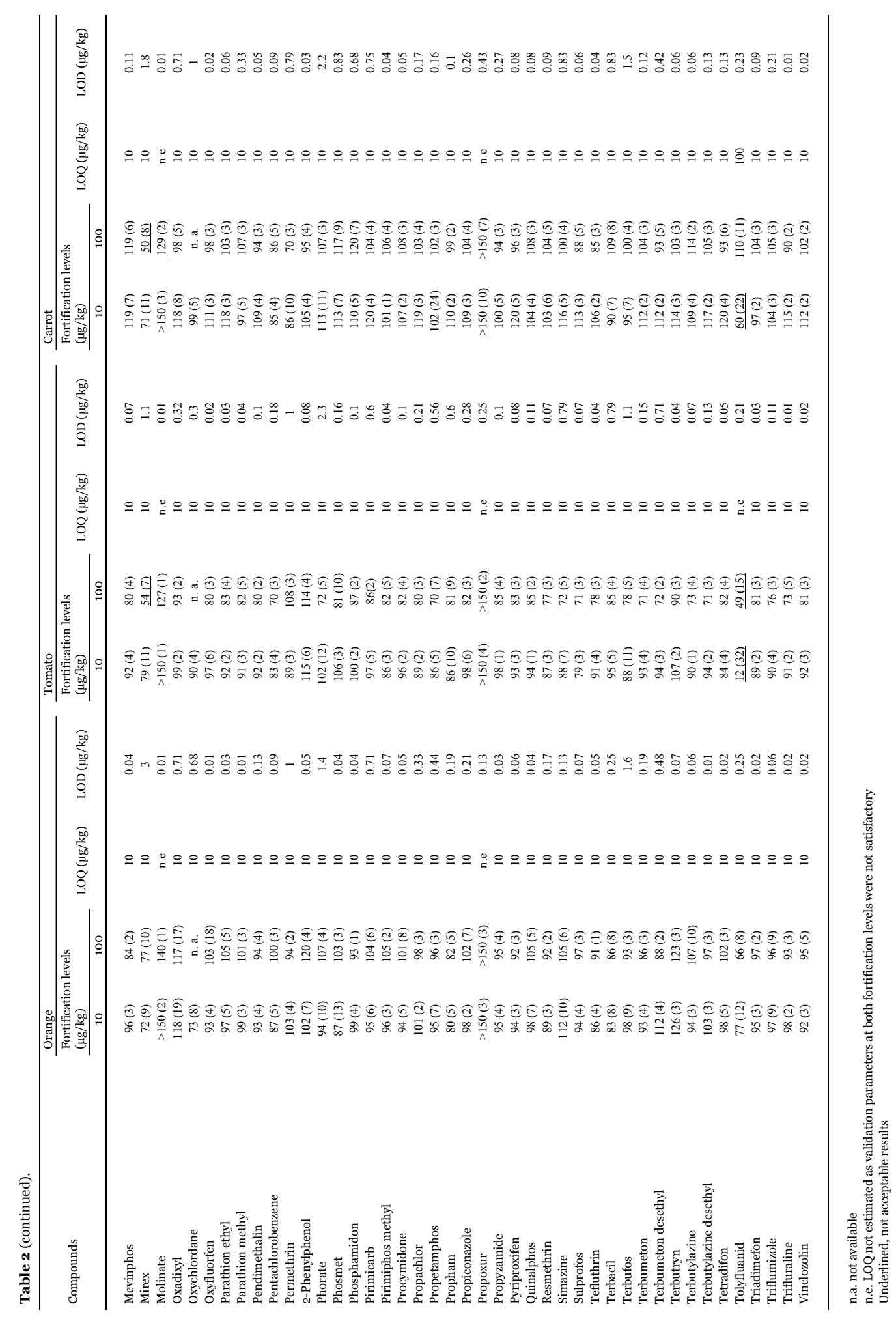

20 

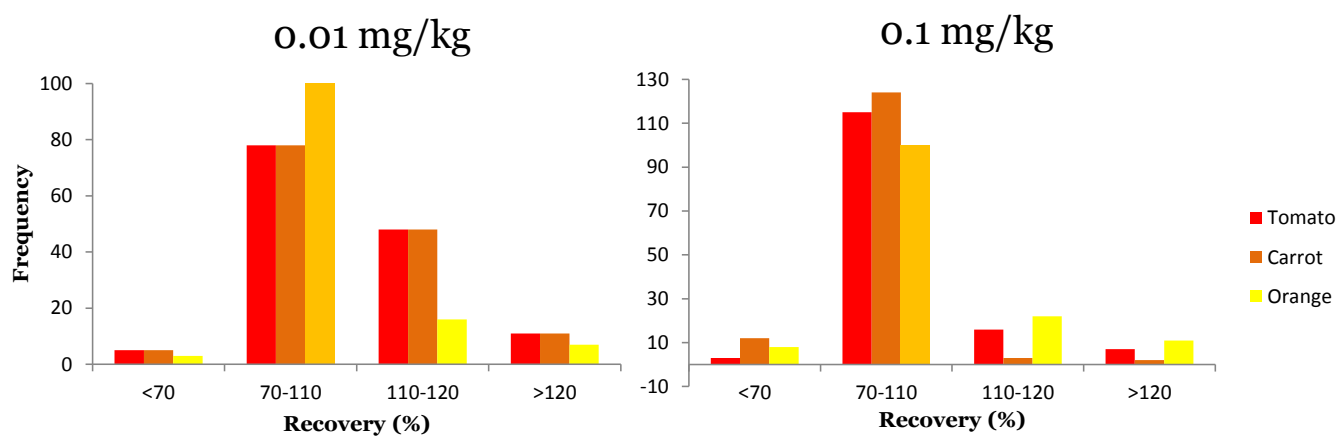

Fig. 1. Histograms obtained from the recovery experiments of the three sample matrices fortified at (a) $0.01 \mathrm{mg} / \mathrm{kg}$ and (b) $0.1 \mathrm{mg} / \mathrm{kg}$.

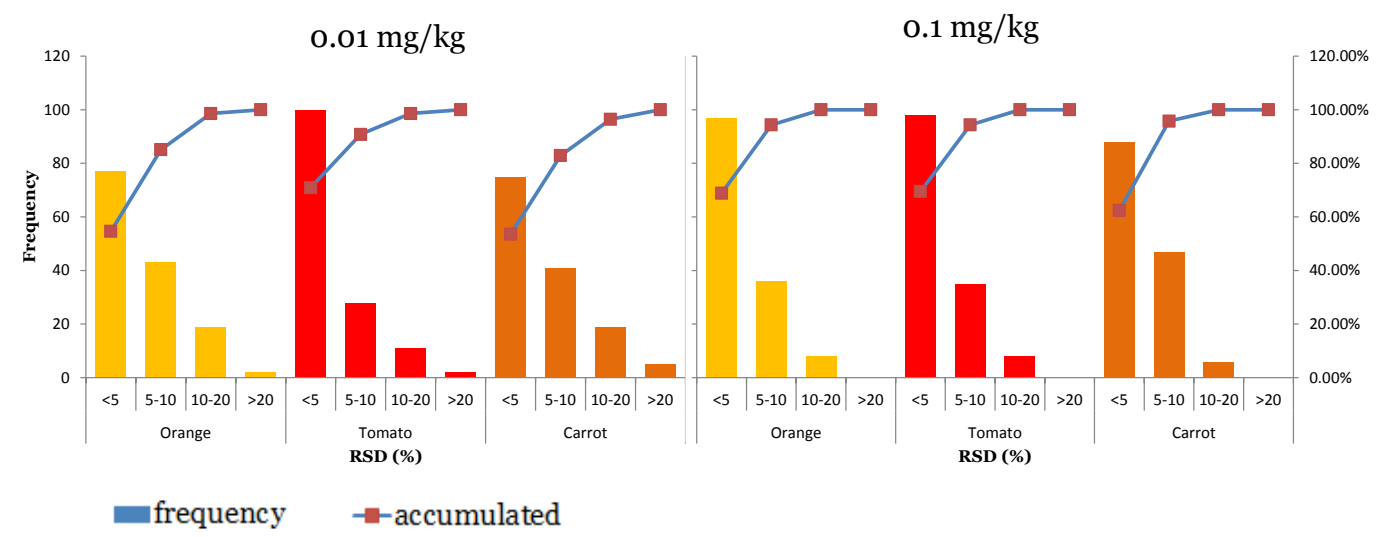

Fig. 2. Histograms obtained from the RSD values of the three sample matrices fortified at (a) $0.01 \mathrm{mg} / \mathrm{kg}$ and (b) $0.1 \mathrm{mg} / \mathrm{kg}$.

Low LODs were obtained for all compounds since most of them ranged between 0.01 and $1 \mathrm{~g} / \mathrm{kg}$ in the three matrices (see Fig. 3). Only few values were higher than $1 \mathrm{~g} / \mathrm{kg}$. Fig. 4 shows four examples (selected from different LOD ranges showed in Fig. 3) for which signal-to-noise ratios were calculated from the lowest matrix-matched standard in orange samples and where LODs can be estimated by extrapolation. 


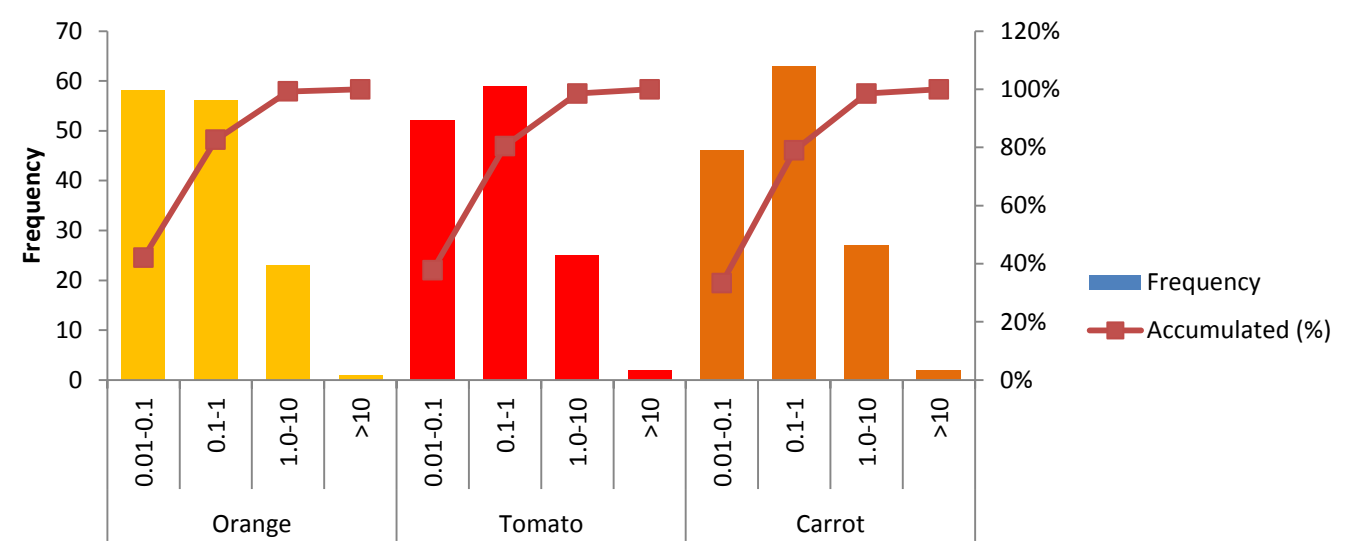

LODs $(\mu \mathrm{g} / \mathrm{kg})$

Fig. 3. Histograms obtained from the LOD values of the three sample matrices.
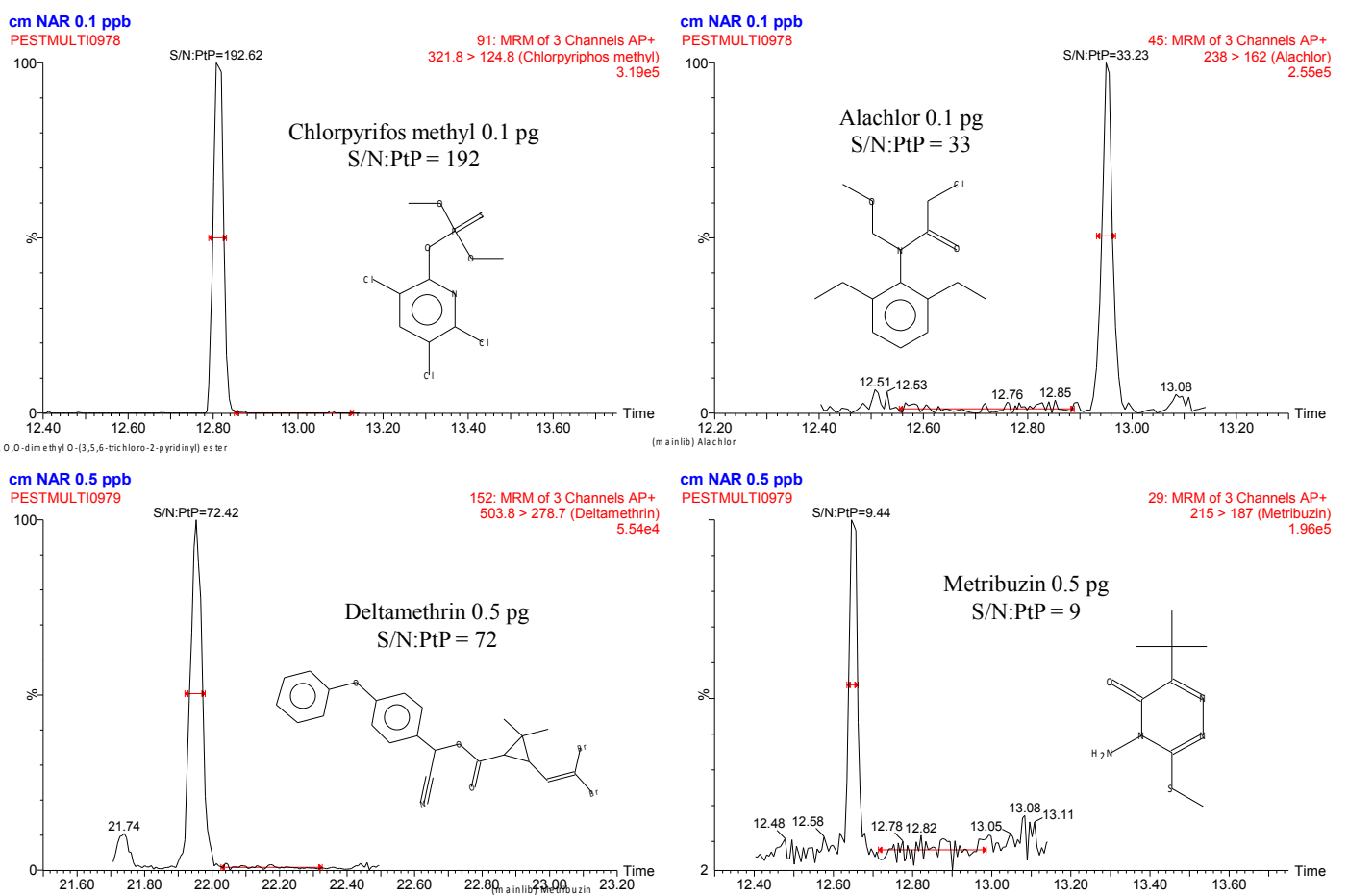

Fig. 4. GC-(APCI) MS/MS chromatogram of four pesticides from the lowest matrix-matched standard (0.1-0.5 ng/mL, corresponding to $0.1-0.5 \mathrm{pg}$ on column) in orange samples. S/N:PtP: peak-to-peak signal-to-noise ratio.

The selectivity, as evaluated for each of the three specific SRM transitions measured, was satisfactory. GC-MS/MS chromatograms did not show interfering peaks at the analyte retention time for any of the pesticides investigated in this work. 


\subsection{Qualitative aspects: consistency of ion ratios and identification}

With respect to the identification of pesticides in samples, criteria have been set for the ratio of the response obtained for the transitions measured [24]. Depending on the relative abundance of the two transitions, the ion ratio should be within $20-50 \%$ of the reference value. This aspect was evaluated in the validation for all pesticides, in each of the three matrices, at the two concentration levels. For each pesticide, two ion ratios were calculated: the first qualifier/quantifier (q1/Q) and the second qualifier/quantifier (q2/Q). The average ion ratio obtained for up to eight matrixmatched standards in the range of $0.1-100 \mathrm{ng} / \mathrm{mL}$ was used as reference ion ratio (values are included in Table 3). For the calculation of the average, signals with poor $\mathrm{S} / \mathrm{N}$ and saturated signals were excluded. In general, the ion ratios for the different concentrations of the standards were very consistent (RSD $<10 \%$ in most cases), even when the ion ratio was very unfavorable $(<0.10)$.

For the spiked samples, the deviation of the individual ion ratios were calculated against the reference value and then compared with the maximum tolerable deviations according to the SANCO guideline [24]. In Table 3, for each pesticide, in each matrix and for each level (with $n=6$ ), the number of ion-ratio compliances is given. Overall, the percentage of pesticides that met the ion ratio criterion for one ratio was $77-81 \%$ at 0.01 $\mathrm{mg} / \mathrm{kg}$, and $95-97 \%$ at $0.1 \mathrm{mg} / \mathrm{kg}$, with not many differences between the three matrices tested. For $60-65 \%$ of the pesticides, the criterion was met for both ratios determined. The reason for not meeting the criteria generally was a too low sensitivity of one of the qualifier transitions measured. For the pesticide methidathion, no suitable qualifier transitions could be obtained and no adequate identification was possible. 


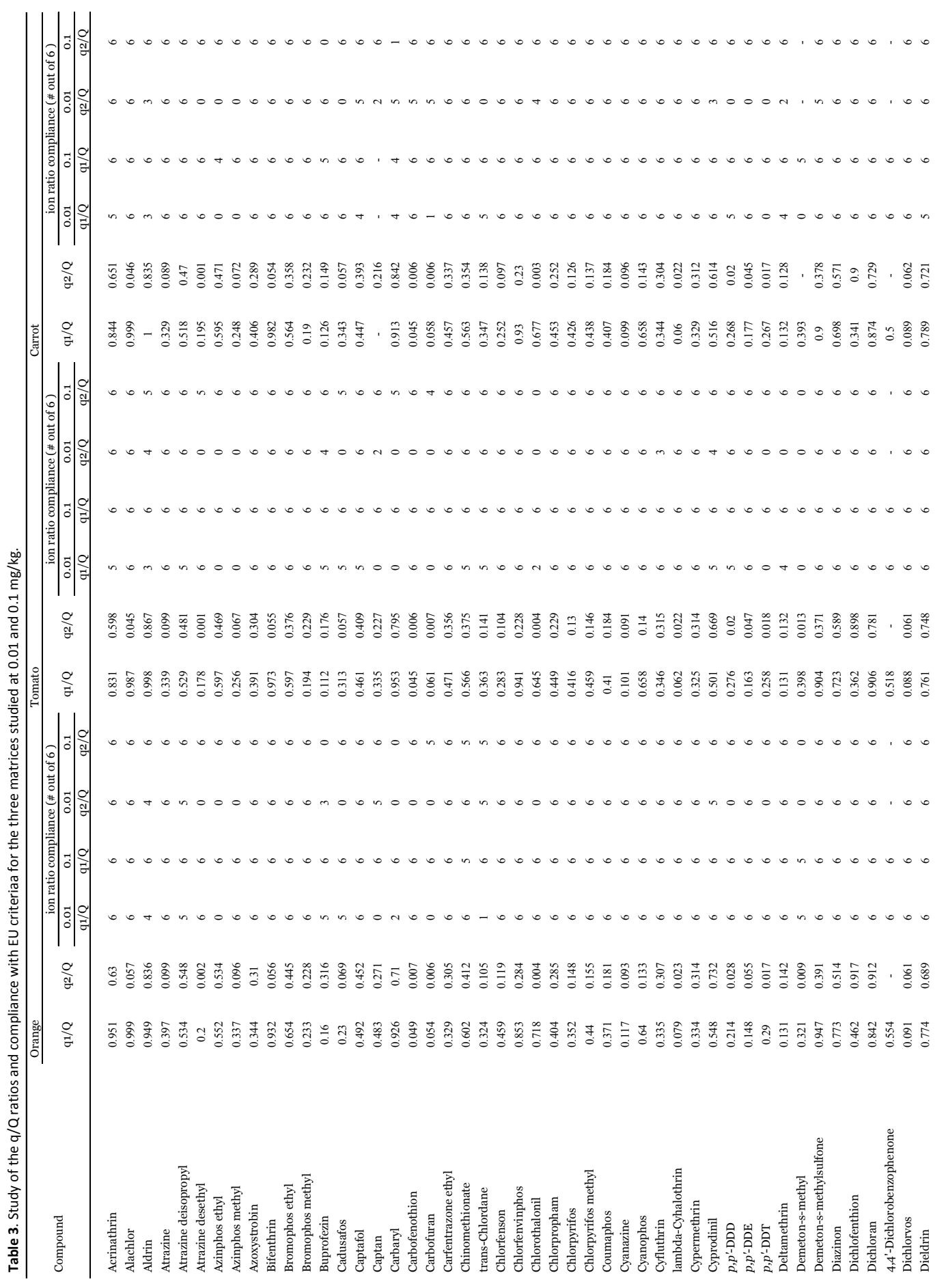




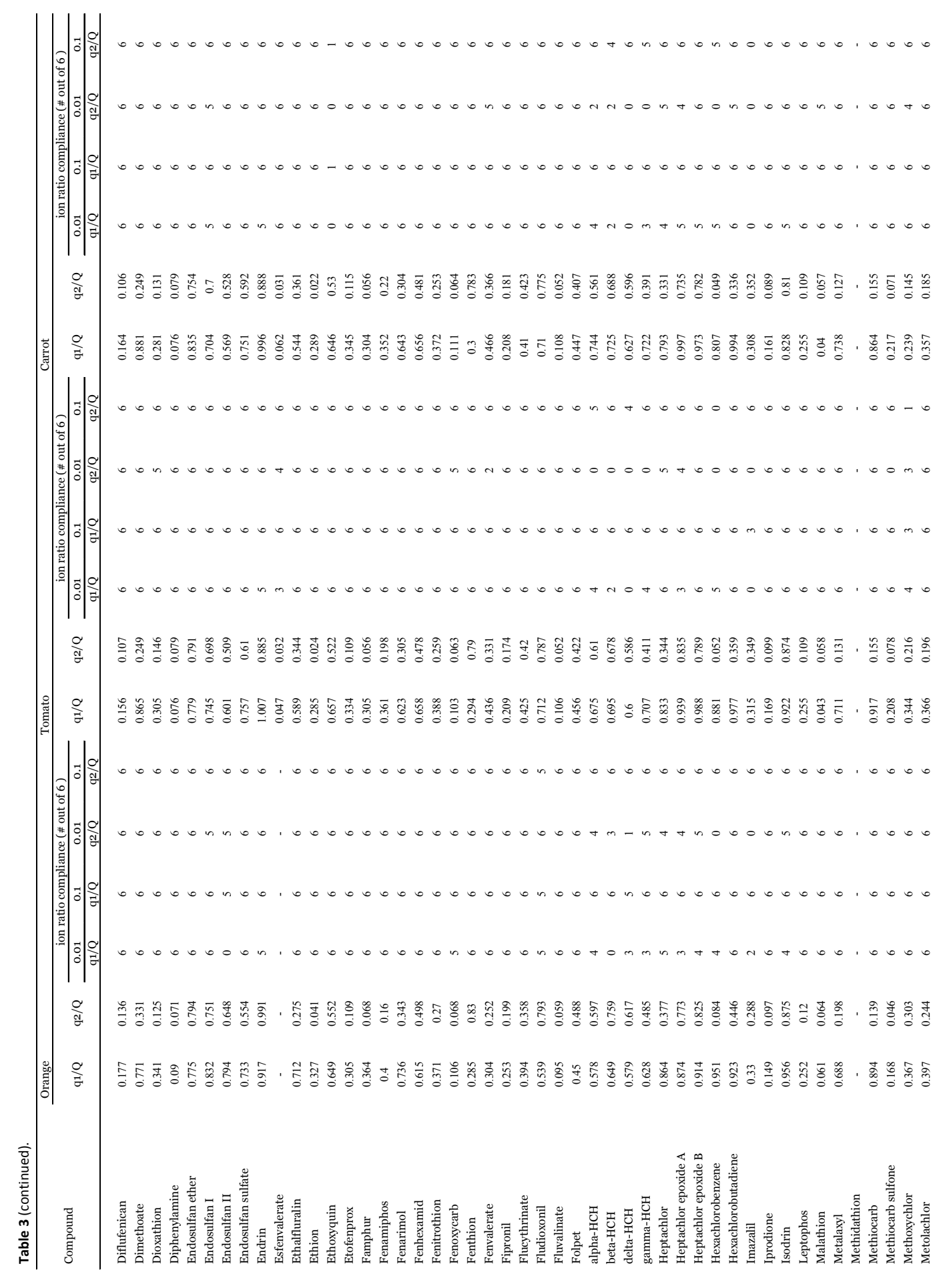




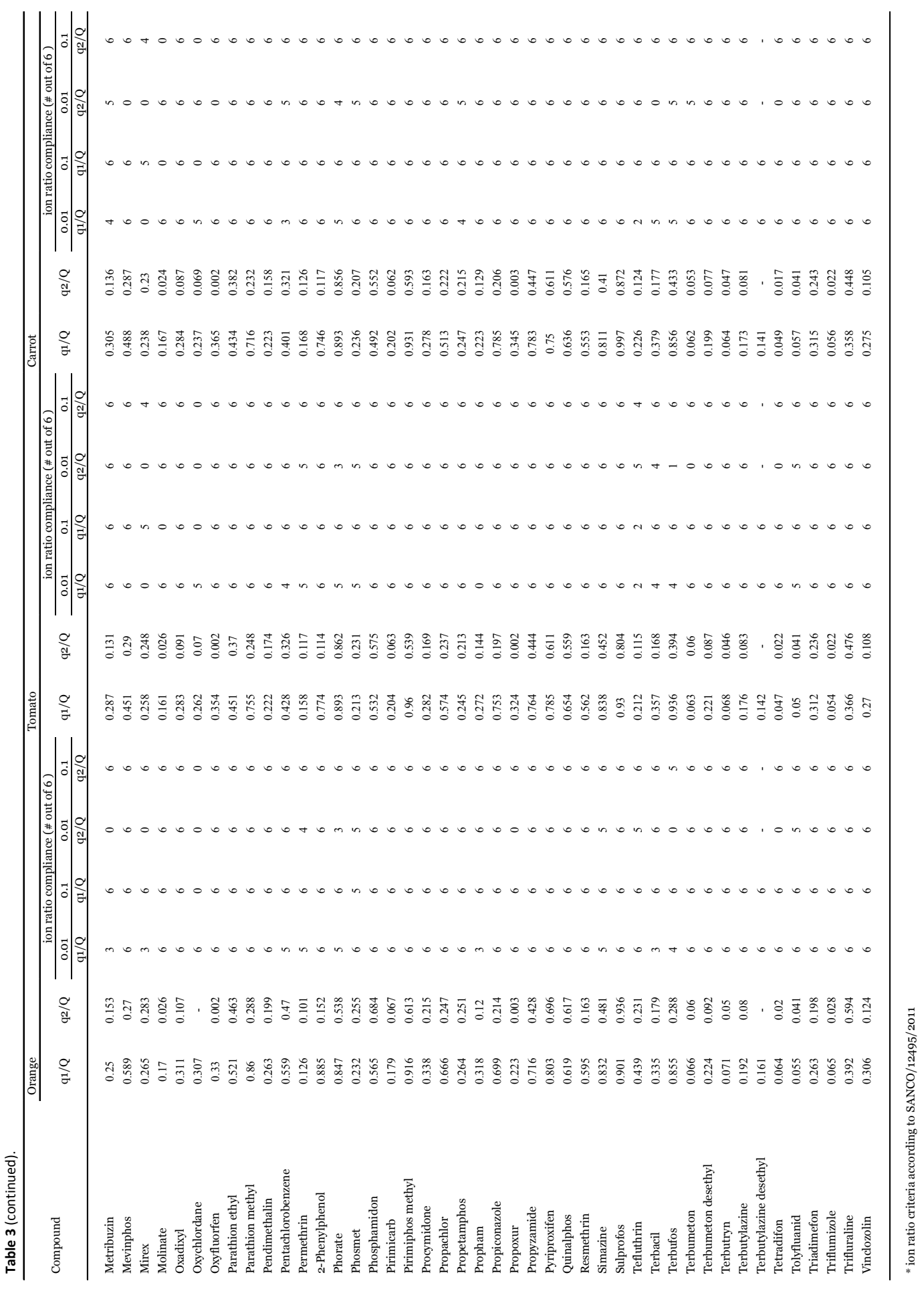




\subsection{Application to real samples}

In order to test the applicability of the developed method, three types of orange, tomato and carrot samples collected from local markets were analyzed. Moreover, the method was expanded for the analysis of three types of apple, lettuce and courgette, including a matrix-matched calibration for each sample matrix and a quality control at 0.05 $\mathrm{mg} / \mathrm{kg}$.

A total of 43 different pesticides were identified in the analyzed samples, most of them at levels well below $0.01 \mathrm{mg} / \mathrm{kg}$ and all under their corresponding MRLs. An overview of the detected pesticides is shown in Table 4.

Orange was the most contaminated sample and several positive findings were present in all the varieties analyzed. In tomato and carrot samples, pesticides were frequently detected but most of them below the LOQ. The different varieties of apple, lettuce and courgette did not present many positive findings, although those in apple samples were the most abundant. Among positive findings, only a small number were found above the LOQ (see Table 5). A concentration level around $1 \mathrm{mg} / \mathrm{kg}$ of the fungicide folpet was the most significant finding, detected in one of the apple varieties, although not exceeding its MRL (3 $\mathrm{mg} / \mathrm{kg}$ ). Captan and bifenthrin, which are commonly used in agricultural crops, were also detected at high levels in apple samples, between 0.1 and $0.5 \mathrm{mg} / \mathrm{kg}$. The OP insecticide chlorpyrifos is also frequently used in apple and orange crops, for which concentrations between 0.03 and $0.1 \mathrm{mg} / \mathrm{kg}$ were found. The maximum positive findings in tomato samples were for the fungicide iprodione (around $0.1 \mathrm{mg} / \mathrm{kg}$ ), whose presence is common in vegetable crops. The higher concentrations levels of pesticides found in carrot samples occurred for metalaxyl and cypermethrin around $0.1 \mathrm{mg} / \mathrm{kg}$. Regarding courgette samples analyzed, no pesticides above $0.01 \mathrm{mg} / \mathrm{kg}$ were found. 
Table 4. List of detected pesticides in the different samples analyzed. Red color indicates the presence of the pesticide in the three varieties of the studied matrix and purple and green, the presence in two and one varieties, respectively.

\begin{tabular}{|c|c|c|c|c|c|c|}
\hline Pesticide & Orange & Tomato & Carrot & Apple & Lettuce & Courgette \\
\hline \multicolumn{7}{|l|}{ Diphenylamine } \\
\hline \multicolumn{7}{|l|}{ Chlorpropham } \\
\hline \multicolumn{7}{|c|}{ Terbumeton desethyl } \\
\hline \multicolumn{7}{|c|}{ Terbutylazine desethyl } \\
\hline \multicolumn{7}{|l|}{ Dimethoate } \\
\hline \multicolumn{7}{|l|}{ Terbutylazine } \\
\hline \multicolumn{7}{|l|}{ Chlorothalonil } \\
\hline \multicolumn{7}{|l|}{ Phosphamidon } \\
\hline \multicolumn{7}{|c|}{ Chlorpyrifos methyl } \\
\hline \multicolumn{7}{|l|}{ Metalaxyl } \\
\hline \multicolumn{7}{|c|}{ Methiocarb sulfone } \\
\hline \multicolumn{7}{|l|}{ Methiocarb } \\
\hline \multicolumn{7}{|l|}{ Chlorpyrifos } \\
\hline \multicolumn{7}{|l|}{ Triadimefon } \\
\hline \multicolumn{7}{|c|}{ 4,4-Dichlorobenzophenone } \\
\hline \multicolumn{7}{|l|}{ Cyprodinil } \\
\hline \multicolumn{7}{|l|}{ Pendimethalin } \\
\hline \multicolumn{7}{|l|}{ Fipronil } \\
\hline \multicolumn{7}{|l|}{ Captan } \\
\hline \multicolumn{7}{|l|}{ Folpet } \\
\hline \multicolumn{7}{|l|}{ Procymidone } \\
\hline \multicolumn{7}{|l|}{ Trifumizole } \\
\hline \multicolumn{7}{|l|}{ Fenamiphos } \\
\hline \multicolumn{7}{|l|}{ Fludioxonil } \\
\hline$p, p^{\prime}-\mathrm{DDE}$ & & & & & & \\
\hline Oxadixyl & & & & & & \\
\hline Sulprofos & & & & & & \\
\hline Famphur & & & & & & \\
\hline Propiconazole & & & & & & \\
\hline Endosulfan su & & & & & & \\
\hline Fenhexamid & & & & & & \\
\hline Propiconazole & & & & & & \\
\hline Diflufenican & & & & & & \\
\hline Iprodione & & & & & & \\
\hline Phosmet & & & & & & \\
\hline Bifenthrin & & & & & & \\
\hline Pyriproxifen & & & & & & \\
\hline Fenarimol & & & & & & \\
\hline Coumaphos & & & & & & \\
\hline Cypermethrin & & & & & & \\
\hline Deltamethrin & & & & & & \\
\hline Azoxystrobin & & & & & & \\
\hline
\end{tabular}


Table 5. Concentrations of pesticides above the LOQ $(\mathrm{mg} / \mathrm{kg})$ detected in analyzed samples.

\begin{tabular}{|c|c|c|c|c|c|c|c|c|c|c|c|c|c|c|c|}
\hline \multirow{2}{*}{ Pesticide } & \multicolumn{3}{|c|}{ Orange } & \multicolumn{3}{|c|}{ Tomato } & \multicolumn{3}{|c|}{ Carrot } & \multicolumn{3}{|c|}{ Apple } & \multicolumn{3}{|c|}{ Lettuce } \\
\hline & S1 & S2 & $\mathrm{S}_{3}$ & S1 & S2 & $\mathrm{S}_{3}$ & S1 & S2 & $\mathrm{S}_{3}$ & S1 & S2 & S3 & S1 & S2 & $\mathrm{S}_{3}$ \\
\hline Azoxystrobin & & & & & & & & 0.023 & & & & & & & \\
\hline Bifenthrin & & & & & & & & & & 0.11 & & & & & \\
\hline Captan & & & & & & & & & & 0.12 & 0.44 & & & & \\
\hline Chlorpyrifos & & 0.11 & 0.035 & & & & & & & 0.028 & 0.042 & 0.059 & & & \\
\hline Chlorpyrifos methyl & & & 0.013 & & & & & & & & & & & & \\
\hline Cypermethrin & & & & & & & & & 0.14 & & & & & & \\
\hline Cyprodinil & & & & 0.015 & & & & & & & & & & & \\
\hline p,p'-DDE & & & & & & & & 0.035 & & & & & & & \\
\hline Fenhexamid & & & & & 0.013 & & & & & & & & & & \\
\hline Fludioxonil & & & & 0.011 & & & & & & & & & & & \\
\hline Folpet & & & & & & & & & & & 1.3 & & & & \\
\hline Iprodione & & & & 0.13 & 0.048 & 0.055 & & & & & & & & & \\
\hline Metalaxyl & & & & & & & & & 0.13 & & & & 0.013 & & \\
\hline Pyriproxifen & & & & 0.024 & & & & & & & & & & & \\
\hline
\end{tabular}

As regards identification, all detected pesticides were identified by the use of three transitions and the compliance of at least one $\mathrm{q} / \mathrm{Q}$ ratio. Identification was problematic at low levels in a few compounds due to unfavorable $\mathrm{q} / \mathrm{Q}$ ratios.

As an illustrative example, Fig. 5 shows GC-(APCI) MS/MS chromatograms corresponding to three of the positive findings detected in analyzed samples: chlorpyriphos in apple $(0.04 \mathrm{mg} / \mathrm{kg})$, pyriproxyfen in tomato $(0.02 \mathrm{mg} / \mathrm{kg})$ and triadimefon in lettuce (below LOQ). A reliable identification of analytes in these samples was feasible by means of the experimental $\mathrm{q} / \mathrm{Q}$ intensity ratios, even at those low concentration levels. 

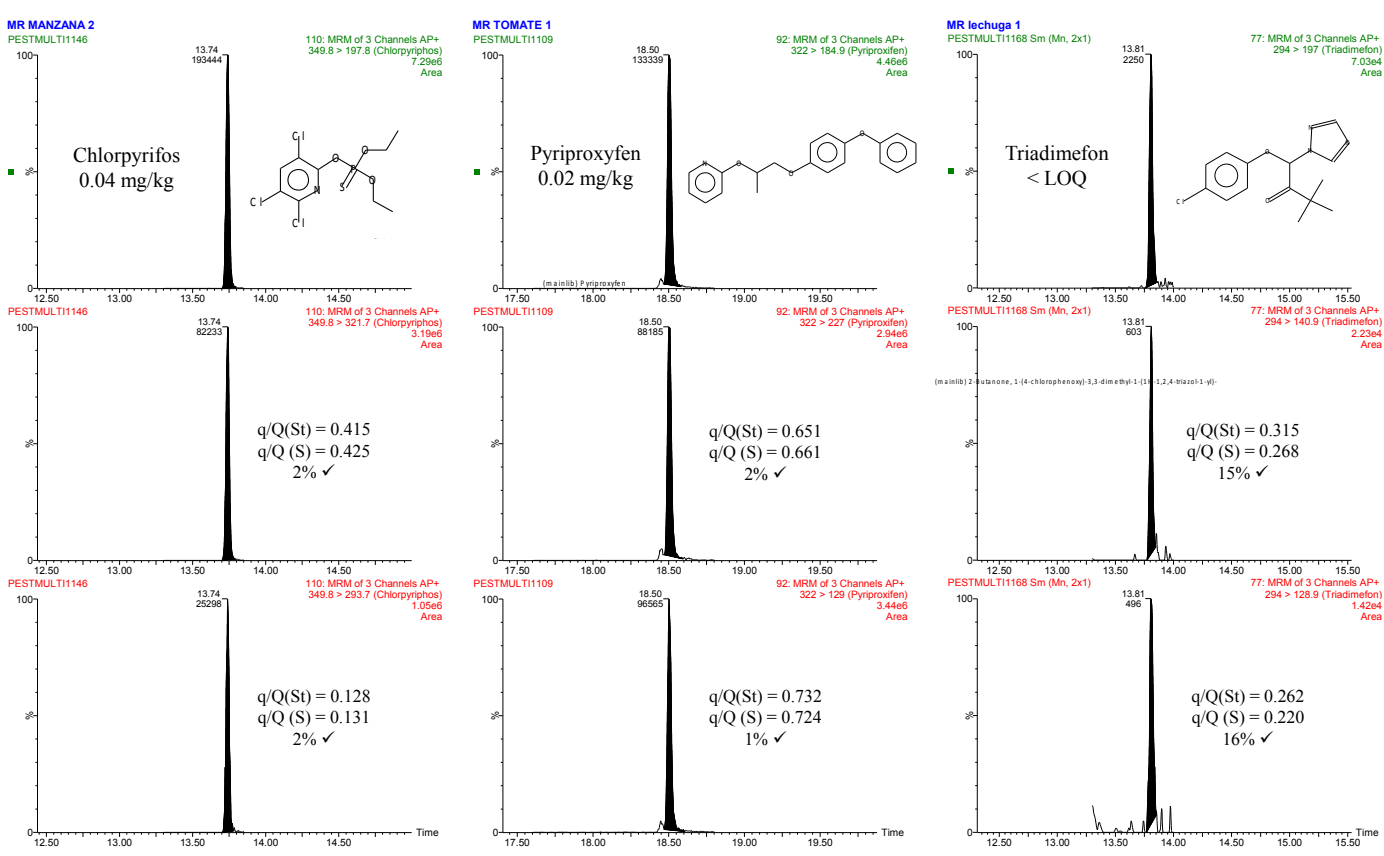

Fig. 5. GC-(APCI) MS/MS chromatograms for pesticides detected in apple, tomato and lettuce. (Q) quantification transition; (q) qualifier transition; (St) standard; (S) sample.

\section{CONCLUSIONS}

A multi-residue method for the determination of pesticide residues in fruit and vegetables was developed with satisfactory results using an innovative system based on an APCI source coupled to GC-(QqQ) MS/MS. The soft ionization allowed the use of the quasi-molecular ion as precursor in most cases contributing to an excellent selectivity and sensitivity. The high sensitivity (LODs of 1-100 fg on-column for most compounds) allowed dilution of QuEChERS extract by a factor of 10, without compromising method detection limits for most of the pesticides studied. The method was successfully validated for the simultaneous quantification and identification of 142 pesticides (three transitions each) in orange, tomato and carrot matrices at 0.01 and $0.1 \mathrm{mg} / \mathrm{kg}$. This demonstrates the suitability of GC-(APCI) MS/MS for quantitative routine residue analysis. Analysis of fruit and vegetable samples allowed identifying and quantifying several pesticides like folpet, captan, bifenthrin, chlorpyrifos, iprodione and chlorothalonil. In all cases, the concentration levels were below the MRLs set by the EU. 


\section{Acknowledgments}

This work has been developed under financial support of Bancaixa (P1-1B2009-25). The authors acknowledge the financial support of Generalitat Valenciana, as research group of excellence PROMETEO/2009/054, and are very grateful to the Serveis Centrals d'Instrumentació Científica (SCIC) of the University Jaume I for the use of GC(APCI)(QqQ) MS/MS Xevo TQ-S. L. Cherta is grateful to University Jaume I for his predoctoral grant and T. Portolés is very pleased to Conselleria de Educación, Formación y Empleo for her postdoctoral grant.

\section{References}

[1] M. Anastassiades, S.J. Lehotay, D. Štajnbaher, F.J. Schenck, J. AOAC Int., 86 (2003), p. 412

[2] S.J. Lehotay, K. Maštovská, A.R. Lightfield, J. AOAC Int., 88 (2005), p. 615

[3] S.C. Cunha, J.O. Fernandes, J. Chromatogr. A, 1218 (2011), p. 7748

[4] S. Walorczyk, J. Chromatogr. A, 1208 (2008), p. 202

[5] L. Cherta, J. Beltran, F. López, F. Hernández, Food Anal. Methods, 6 (2013), p. 1170

[6] L. Alder, K. Greulich, G. Kempe, B. Vieth, Mass Spectrom. Rev., 25 (2006), p. 838

[7] L. Cherta, J. Beltran, T. Portolés, F. Hernández, Anal. Bioanal. Chem., 402 (2012), p. 2301

[8] P. Zhao, L. Wang, L. Zhou, F. Zhang, S. Kang, C. Pan, J. Chromatogr. A, 1225 (2012), p. 17

[9] D.I. Kolberg, O.D. Prestes, M.B. Adaime, R. Zanella, Food Chem., 125 (2011), p. 1436

[10] M.I. Cervera, C. Medina, T. Portolés, E. Pitarch, J. Beltrán, E. Serrahima, L. Pineda, G. Muñoz, F. Centrich, F. Hernández, Anal. Bioanal. Chem., 397 (2010), p. 2873

[11] C.M. Medina, E. Pitarch, T. Portolés, F.J. López, F. Hernández, J. Sep. Sci., 32 (2009), p. 2090

[12] P.P. Bolaños, J.L.F. Moreno, D.D. Shtereva, A.G. Frenich, J.L.M. Vidal, Rapid Commun. Mass Spectrom., 21 (2007), p. 2282 
[13] J. Robles-Molina, B. Gilbert-López, J.F. García-Reyes, N.R. Martos, A. Molina-Díaz, Anal. Methods, 3 (2011), p. 2221

[14] F.J. Camino-Sánchez, A. Zafra-Gómez, S. Cantarero-Malagón, J.L. Vílchez, Talanta, 89 (2012), p. 322

[15] A. Amirav, A. Gordin, M. Poliak, A.B. Fialkov, J. Mass Spectrom., 43 (20o8), p. 141

[16] E.C. Horning, D.I. Carroll, I. Dzidic, Clin. Chem., 23 (1977), p. 13

[17] R. Schiewek, M. Lorenz, R. Giese, K. Brockmann, T. Benter, S. Gäb, O.J. Schmitz, Anal. Bioanal. Chem., 392 (2008), p. 87

[18] T. Bristow, M. Harrison, M. Sims, Rapid Commun. Mass Spectrom., 24 (2010), p. 1673

[19] R. García-Villalba, T. Pacchiarotta, A. Carrasco-Pancorbo, A. Segura-Carretero, A. Fernández-Gutiérrez, A.M. Deelder, O.A. Mayboroda, J. Chromatogr. A, 1218 (2011), p. 959

[20] A. Carrasco-Pancorbo, E. Nevedomskaya, T. Arthen-Engeland, T. Zey, G. Zurek, C. Baessmann, A.M. Deelder, O.A. Mayboroda, Anal. Chem., 81 (2009), p. 10071

[21] T. Portolés, J.V. Sancho, F. Hernández, A. Newton, P. Hancock, J. Mass Spectrom., 45 (2010), p. 926

[22] T. Portolés, J.G.J. Mol, J.V. Sancho, F. Hernández, Anal. Chem., 84 (2012), p. 9802

[23] T. Portolés, L. Cherta, J. Beltran, F. Hernández, J. Chromatogr. A, 1260 (2012), p. 183

[24] European Control Guidelines SANCO/12495/2011.

[25] T. Cajka, K. Maštovská, S.J. Lehotay, J. Hajšlová, J. Sep. Sci., 28 (2005), p. 1048

[26] S.J. Lehotay, A. De Kok, M. Hiemstra, P. Van Bodegraven, J. AOAC Int., 88 (2005), p. 595

[27] A. Peruga, M. Barreda, J. Beltrán, F. Hernández, Food Addit. Contam. Part A: Chem. Anal. Control Expo. Risk Assess., 30 (2013), p. 298 